\title{
1 Horizontal transfer of a conserved npc-2 like 2 effector gene in rust fungi that suppresses cell 3 death in plants
}

4 Rajdeep Jaswal1, 2, Himanshu Dubey4, Kanti Kiran4, Hukam Rawal4, Gulshan Kumar1,

5 Sivasubramanian Rajarammohan ${ }^{1}$, Rupesh Deshmukh ${ }^{1}$, Humira Sonah ${ }^{1}$ Pramod Prasad3,

6 Subhash C Bhardwaj ${ }^{3,}{ }^{*}$ Naveen Gupta ${ }^{2,}$ " Tilak Raj Sharma ${ }^{1,4}$

7 Affiliations:

$8 \quad{ }^{1}$ National Agri-Food Biotechnology Institute (NABI), Mohali, Punjab, India -140306

9 2Department of Microbiology, Panjab University, Chandigarh, Punjab, India - 160014,

10 3ICAR-Indian Institute of Wheat and Barley Research, Regional Station, Flowerdale,

11 Shimla, India - 171009,

12 4National Institute on Plant Biotechnology, Pusa Campus New Delhi, India - 110012

\section{Corresponding author}

Correspondence to: Dr. T R Sharma (trsharma1965@gmail.com ),

Phone: 91-172-4990101

17 National Agri-Food Biotechnology Institute (NABI),

Mohali, Punjab, India -140306

Correspondence to: Dr. Naveen Gupta (ng.puchd@gmail.com)

21 Phone: 91-9872692684

Department of Microbiology, Panjab University,

23 Chandigarh, Punjab, India - 160014 


\section{Abstract}

ML/MD-2 is a conserved lipid/sterol-binding protein family having a role in sterol transfer and innate immunity in lower and higher eukaryotes. Here we report a genome-wide survey of this family, identifying 84 genes in 25 fungal and five oomycetes plant pathogen, having a different nutrition mode. All the fungal species were found to have varied numbers of family members, a distinctively substantial expansion of the ML gene family was observed in Rhizophagus irregularis (RI) with 33 genes. Our analysis also showed that NPC2 like proteins, a subfamily of ML domain superfamily, were not only restricted to animals and insect species but also present in plant fungal pathogens, including members of Clavicipitaceae, Pucciniacease, and Tremellaceae family. The phylogenetic analysis showed that these NPC2 like fungal proteins are more closely related to animals/insects than other fungal species. The molecular docking studies of these proteins with cholesterol and other derivatives indicate lipid-binding functional conservation across the animal and fungi kingdom. Further, the full length CDS of one of the npc2 like genes from Puccinia triticina (Pt5643) was PCR amplified and further characterized using various studies such as qRT-PCR, expression in onion epidermal cells, Nicotiana benthamiana for subcellular localization studies, yeast functional complementation, and expression studies. The mRNA abundance of Pt5643 was observed to be increased along with the infection progression and exhibits the highest expression at 5 thday post-infection (dpi), suggesting its important role in the $P$. triticina infection cycle in wheat. The fluorescent confocal microscopy of transiently expressed YFP tagged Pt5643 in onion epidermal cells and N. benthamiana shows its location in cytoplasm and nucleus, indicating its involvement in the manipulation of host genes. The functional complementation of Pt5643 in npc2 mutant 
48 yeast showed its functional similarity to the eukaryotic npc2 gene. Further, the

49 overexpression of Pt5643 also suppressed the BAX and H2O2 induced program cell death

50 in $N$. benthamiana and yeast, respectively thus proving to be a novel horizontally

51 transferred effector in rust fungal pathogens. Altogether the present study reports the

52 novel function of fungal NPC2 like proteins playing a crucial role in host defense

53 manipulation possibly through lipid binding/transport similar to animals.

\section{$54 \quad$ Keywords}

55 Rust fungi; plant cell death; effector proteins; horizontal gene transfer; ML domain

\section{Introduction}

57 In nature, plants and pathogens interact with each other continuously. Both host and pathogen need myriads of small molecules like carbohydrates, proteins, lipids for various cellular processes. Out of these, lipids and sterols perform crucial roles in various processes ranging from making cell membranes to function as defense signaling molecules. Sterols constitute major components in the cell membrane of the eukaryotic cell. The presence of various sterols in different proportions constitutes the variability of cell membranes in all eukaryotes.

The effector proteins are one of the essential arsenals of fungal pathogens including rusts to disarm the host(Jaswal et al., 2020a, 2019). Most of the rust effectors are highly hostspecific and do not show any similarity to known domain proteins(Jaswal et al., 2020b).

67 Several effectors need to possess a conserved domain or fold to perform a function as an effector. The host genes' evolutionary selection pressure causes most of the effector genes to changes their sequences. In this scenario, the pathogens change these gene sequences; 
however, they may maintain the minimum conserved fold to perform a corresponding

71 biological function. Moreover, the pathogen may also generate duplicate copies to perform

72 the function to minimize the selection pressure on the single gene. In the other case, the

73 pathogen may also acquire certain genes from the environment to attain novel traits to

74 deceive the host in infection pressure.

75 The horizontal gene transfer is also known as lateral gene transfer, is a common method of acquiring foreign genes by organisms from other species. In the case of fungal organisms,

77 various studies have reported the presence of HGT genes donated by bacterial as well as other eukaryotic organisms like fungus, plants, and other species (Coelho et al., 2013; Fitzpatrick, 2012; Qiu et al., 2016; Slot and Rokas, 2011; Yin et al., 2016). However, relatively few studies have reported the inter-eukaryotic gene transfer.

MD-2-related lipid-recognition (ML) is one of the protein domain superfamily present in eukaryotes (plants, animals, fungi, protists) having lipid and sterol binding activity. ML superfamily includes $\mathrm{MD}-1, \quad \mathrm{MD}-2, \quad \mathrm{NPC} 2$ proteins, $\mathrm{GM} 2$ activator, phosphatidylinositol/phosphatidylglycerol transfer protein (PG/PI-TP), and mite allergen Derp2 protein, including several other proteins with unknown functions (Inohara and Nuñez, 2002). The ML Proteins generally contain a single domain (150 amino acids), Nterminal signal peptide, immunoglobulin-like antiparallel beta rich folds, and conserved cysteine residue with hydrophobic pocket present at the center suitable for binding diverse lipid/sterol molecules(Inohara and Nuñez, 2002). Crystallographic structures of various ML family proteins like NPC2, GM2-AP, and Derp2 have been studied and analyzed

91 (Johannessen et al., 2005; Kim et al., 2007; Xu et al., 2007). The crystal structure analysis 92 of bovine NPC2 revealed that the sterol binding site is present inside a deep hydrophobic 
93 pocket between the two beta sheets. The structure analysis also showed the binding site to

94 be compact and small in the absence of ligand, which tends to expand after binding of a sterol ligand (Xu et al., 2007).

ML superfamily genes characterized in organisms such as insects and humans are known

97 to perform diverse functions like providing innate immunity, chemical communication, and sterol trafficking(Frolov et al., 2003; Shi et al., 2012). The Drosophila melanogaster NPC2a and NPC2e proteins have a varied number of cysteine residues and bind with bacterial cell wall components in vitro, providing innate immunity (Shi et al., 2012). The $n p c 2 a$ and $n p c 2 b$ genes in $D$. melanogaster are similar to vertebrate $n p c 2$ genes and regulate steroid biosynthesis and sterol homeostasis (Huang et al., 2007). In Anopheles gambiae, these proteins play an important role in immunity against Plasmodium falciparum (Dong et al., 2006). The ML protein from tobacco hornworm binds to lipopolysaccharide (LPS) and is possibly involved in LPS mediated defense signaling pathways against Gram-negative bacteria (Ao et al., 2008). challenged with LPS (Liao et al., 2011). In addition to this, ML family members are also known to play an important role by acting as agonists in dengue virus infection by modifying mosquito immunity (Jupatanakul et al., 2014). One of the invertebrate ML superfamily members identified in Ciona intestinalis homologous to the vertebrate npc2 gene and is induced after LPS inoculation (Vizzini et al., 2015). In the case of plant fungal pathogens, very few have been explored to characterize the functions of this superfamily.

114 Anpc1 like gene has been reported to perform sterol trafficking in the Fusarium 115 graminearum (Breakspear et al., 2011). Metarhizium robertsii an insect fungal pathogen, 
116 has acquired npc2- like gene from its insect host in the course of evolution through

117 horizontal gene transfer (Zhao et al., 2014). No other study has reported the function of

118 this superfamily in the plant-fungal pathogens

119 In the present study to explore the status of the ML superfamily, the genome-wide 120 identification method has been employed in 25 fungal and five oomycetes species having 121 different modes of nutrition. We have compared these proteins at the sequence level, 122 analyzed the chromosome location, and identified the phylogenetic relationship across 123 eukaryotes (fungi, plants, animals, and protists). The comparison of structures of proteins 124 at the tertiary level three and molecular docking was done to find out the possible 125 interacting partners. Selection pressure studies were also performed. Expression patterns 126 were also studied using the SRA NCBI database as well as the real-time PCR. Subcellular 127 localization was also performed to predict the location of one of the $P$. triticina npc2- like 128 genes.

129 The functional similarity of the rust $m l$ gene Pt5643 was also checked using 130 complementation assay in the npc-2 mutant of the Saccharomyces cerevisiae. Moreover, 131 in-planta and S.cereviseae expression of Pt5643 was done to evaluate its capacity as an 132 effector. This study provides the first report of the presence of $n p c 2$ like genes in rust fungi 133 and further its role as an effector. Moreover, the study also provides a baseline for exploring the ML superfamily in eukaryotes Additionally, it can also be utilized to elucidate 135 the evolutionary relationship of the ML gene family across plant fungal pathogens and 136 predict its functional importance as an effector. 


\section{Materials and methods}

\subsection{Identification of $\mathrm{ml} / \mathrm{md}-2 g e n e s$ across fungal pathogens}

140 The fungal proteomes were downloaded from the UniProt database

141 (http://www.uniprot.org/help/uniprotkb) and NCBI (https://www.ncbi.nlm.nih.gov).

142 The predicted protein sequences of Puccinia triticina and Puccinia strïformis used for this study were taken from the data generated by Kiran et al., 2016 and 2017 (Kiran et al., 2017, 2016). All the putative ML/MD-2 domain-containing proteins were retrieved from the Interpro database (https://www.ebi.ac.uk/interpro/entry/IPRo031672). The psi-blast program was used to perform genome-wide identification using downloaded ML/MD-2 proteins (IPRo33916) as a query against all the organisms (Altschul et al., 1990). Target proteins with BLAST hit (E-value<-5) were selected and subjected to NCBI-CD search (https://www.ncbi.nlm.nih.gov/Structure/cdd/wrpsb.cgi) and InterPro database (http://www.ebi.ac.uk/interpro/search/sequence-search) for conserved domain analysis.

151 Redundant and nonspecific hit-containing proteins were filtered out, and proteins with 152 ML/MD-2 domain-containing proteins were selected as candidate proteins.

\section{physicochemical properties analyses}

155 Secretory protein analysis was done by using SignalP 3.0 for signal peptide analysis

156 (http://www.cbs.dtu.dk/services/SignalP/). TargetP1.1 and CELLO were used to analyze

157 the subcellular localization of genes. (http://www.cbs.dtu.dk/services/TargetP/)

158 , (http://cello.life.nctu.edu.tw/). The TMHMMv2.o was used for finding transmembrane159 containing proteins. (http://www.cbs.dtu.dk/services/TMHMM/). GPI Modification Site 
Prediction in Fungi was predicted using big-PI Fungal Predictor

161 (http://mendel.imp.ac.at/gpi/fungi server.html). The amino-acid and atomic

162 compositions, theoretical pI, the composition of amino acids, the molecular weight of all

163 ML/MD-2 proteins was calculated using the ExPASy server's ProtParam and Compute 164 pI/Mw tool. (http://web.expasy.org/protparam/), (http://web.expasy.org/compute pi/).

165 All the tools were run using default parameters.

2.3. Chromosomal localization and gene structure analysis, Motif and Domain analysis

The chromosomal location of the $m l$ genes was determined for fungal pathogens having chromosomal data in NCBI using Mapchart 2.2. BLASTn was done to retrieve the specific location of genes for the corresponding organism. Gene structures of $\mathrm{ml}$ genes were

171 generated using GSDS (http://gsds.cbi.pku.edu.cn/); motifs were predicted using the

172 MEME program (http://meme.nbcr.net/meme/cgi-bin/meme.cgi). The Parameter

173 selected for the analysis was as follows: total motif predictions were restricted to 10, and

174 the size of the motif was given from 4-50 amino acids. The full-length ML/MD-2 proteins

175 were annotated first using CD-search for different domain identification. The representing 176 structures of various domains were designed using IBS 1.0 (http://ibs.biocuckoo.org/).

\section{2.4. Protein Structure prediction and docking analysis}

$1783 \mathrm{D}$ Structures of all the secretory proteins were predicted using I-Tasser software and 179 further refined by modrefinner(Xu and Zhang, 2011; Yang et al., 2015). Ramachandran 180 plot analysis was done using Rampage software, showing $>80 \%$ amino acids in the core 181 region for all the structures (http://mordred.bioc.cam.ac.uk/ rapper/rampage.php). Final 
182 validation for all the structures was done using tools available on the SAVES server 183 (https://services.mbi.ucla.edu/SAVES/). Docking analysis was done using the Docking 184 Server (Bikadi and Hazai, 2009). Ligands were retrieved from PubChem in sdf format and 185 changed in pdb format with the help of an open babel tool. All the structures were 186 converted into energy minimized states using Hamiltonian- Force Field-MMFF94x (Halgren, 1996). Gasteiger partial charges were added to ligand atoms. AutoDock tool was used to add Kollman united atom type charges, essential hydrogen atoms solvation parameters.

\subsection{Multiple sequence alignment and phylogenetic analysis}

The sequence alignment of ML/MD-2 proteins was done using MAFFT software (online version) (http://www.ebi.ac.uk/Tools/msa/mafft/ ). For comparative analysis, proteins from major host plants of different pathogens and few model species (Rice, Wheat, 194 Tomato, Potato, Maize, Soybean, and Human, insects, protists) were included in the study and aligned. For sequence alignment, the ML/MD-2 domain region was extracted using

196 python script for all the proteins (including host plants and model species). For 197 phylogenetic analysis, full-length proteins were aligned using MAFFT, model testing was 198 done using MEGA7.0 (Kumar et al., 2016), and the best-fitted model was considered for 199 the tree construction. The phylogenic tree was constructed in MEGA7.0 with the maximum 200 likelihood (ML) method with 1000 bootstrap replicates. A total of 68 different eukaryotes 201 (Fungi-22, Plants-6, oomycetes-5, protists-28, animals-5) were used in the analysis to infer 202 the phylogenetic relationship of ML proteins among these species. 
204

205

206

207

208

209

210

211

212

213

214

215

216

217

218

219

220

221

222

223

224

225

The nonsynonymous substitutions (Ka) and synonymous substitutions (Ks) were utilized to analyze section pressure of the $M L$ genes (Li et al., 1981). DNASP5.0 software was used to analyze the $\mathrm{Ka} / \mathrm{Ks}$ ratios between the genes (Librado and Rozas, 2009). Nucleotide sequences (CDS) were aligned first and used as a query. Results were analyzed on the presumption that purifying selection causes values less than $1(\mathrm{Ka} / \mathrm{Ks}<1)$, and positive selection leads to a higher value than $1(\mathrm{Ka} / \mathrm{Ks}>1)$ (Juretic et al., 2005).

\subsection{Plant materials, pathogen inoculation, tissue harvest, RNA extraction}

\section{and real-time quantitative PCR}

Susceptible wheat variety (Agra Local) was used as a host for infection of $P$. triticina, $P$. striiformis, and B. graminis. Uninfected (Control) and infected leaves were harvested at various time intervals (0,3,5,7 9 and $14 \mathrm{dpi}$ ), respectively, immediately frozen in liquid N2 and stored at $-80^{\circ} \mathrm{C}$ for further analysis. Total RNA was isolated from mirVana ${ }^{\mathrm{TM}}$ miRNA Isolation Kit, Thermo Fisher Scientific (USA).

First-strand cDNA synthesis was done from RNA using the iScript ${ }^{\mathrm{TM}}$ (Biorad) USA reverse transcription kit with approximately $1 \mu \mathrm{g}$ RNA according to the manufacturer's instruction. For qRT-PCR, primers were designed using Oligocalc software and with amplicon lengths of 120-250 bp. The qRT-PCR was done on 7500 ABI Real-time PCR systems (Applied Biosystems, United States) using Taq ${ }^{\mathrm{TM}}$ Universal SYBR® Green Supermix(Biorad) USA. The relative expression was calculated by the $2^{-\Delta \Delta C t}$ method(Livak and Schmittgen, 2001). The PtActin and PtGAPDH were used as internal controls.

\subsection{Cloning, In-vitro, In-planta, and yeast expression}

Amplification of the coding sequence (CDS) Pt5643 gene ( $P$. triticina npc2 like gene) was 
226 done using full length gene-specific primers with BamHI (forward primer) and NotI 227 (reverse primer) restriction sites and cloned into the pGEM-T easy vector. Confirmation 228 of the clone was done through PCR, restriction digestion, and sequencing. The confirmed 229 fragment was further mobilized into the pET 29a (+) vector and transformed into BL21 230 E.coli expression strain; induction for invitro expression was done by using $0.1 \mathrm{mM}$ IPTG 231 at $37{ }^{\circ} \mathrm{C}$ and analyzed on the $12 \%$ SDS-PAGE. For In-planta expression and subcellular 232 localization experiments, full length CDS ( without signal peptide coding region and stop 233 codon ) of Pt5643 and BAX gene (full length CDS PCR amplified from mouse cDNA) was 234 cloned using gateway cloning vector pENTER and further mobilized into the destination 235 pGWB408 for overexpression. For subcellular localization, the pENTER_Pt5643 clone 236 was used for mobilizing the Pt5643 in the pGWB441. Dr. Tsuyoshi Nakagawa's lab 237 generously provided the binary plant transformation vector pGWB408 and pGWB441. 238 For expression in the yeast, the pENTER_Pt5643 clone was used for mobilizing the 239 Pt5643 in the pYESDEST52 gateway destination vector.

\subsection{Subcellular localization of Pt5643 gene}

241 For subcellular localization, onion epidermal cells, particle bombardment system, and a

242 Nicotiana benthamiana agrobacterium mediated transient expression methods were 243 used. The confirmed pGWB4441_Pt5643 construct (YFP) with the C-terminal fluorescent 244 tag and an empty vector containing only fluorescent tag was bombarded separately on 245 onion peels containing MS plates using the PDS100o/He system at 1100 psi (BioRad, 246 USA). After 36 hrs, the samples were visualized under Zeiss LSM 880 with laser scanning 247 confocal microscope (Carl Zeiss Microscopy, USA). 
248 To confirm the subcellular localization of Pt_5643, 0.5 optical density (OD) 249 agrobacterium (strain GV3101) culture containing Pt_5643_pGWB441 and empty vector

250 was infiltrated in separate $N$. benthamiana leaves and analyzed after three days at the 251 infiltrated areas at $514 \mathrm{~nm}$ in the confocal microscope.

\section{2.10 Functional complementation assay in npc2 yeast mutants}

253 For function complementation assay, the wild-type yeasts BY4741 and the npc2 mutant 254 (YDLo46W) were used. The npc2 mutant competent cells were transformed with 255 pYESDEST52 and pYESDEST52_Pt5643 construct separately and further selected on the uracil dropout medium. The functional complementation was analyzed on 100mM acetic acid plates by spotting assay (1-1/1000 dilutions) as the npc2 mutants are resistant to 259 days.

2.11 Bax and $\mathrm{H} 2 \mathrm{O2}$ induced cell death in transient expression in $N$. benthamiana and $S$. cerevisiae transformed in Agrobacterium tumefaciens GV3101 competent cells and further

264 confirmed by PCR. $N$. benthamiana plants grown up to 3-4 weeks were used 265 agroinfiltration. The cultures were adjusted to an optical density (OD) of 0.5 and 266 suspended in MES buffer containing $10 \mathrm{mM} \mathrm{MgCl}$, $10 \mathrm{mM}$ 2-(N-morpholino) 267 ethanesulfonic acid (MES), $\mathrm{pH}$ 5.6, and $100 \mu \mathrm{M}$ acetosyringone. The BAX construct was 268 infiltrated o, 12, and 24 hrs post Pt5643 construct infiltration in different spots. The 269 phenotype was observed after 5-8 days of infiltration. The $\mathrm{H}_{2} \mathrm{O}_{2}$ induced oxidative stress 
270 hijacking phenotype analysis was done using $5 \mathrm{mM} \mathrm{H}_{2} \mathrm{H}_{2}$ containing YPD plates. 271 Approximately $1 / 1000$ cells were plated from the OD 1.0 of yeast cells for $n p c 2$ mutant 272 cells, wild type (BY4741), and pYESDEST52_Pt5643 containing npc2 mutants grown in 273 YPD broth medium. The results were analyzed after 3-4 days.

274

275

276

277

278

279

280

281

282 


\section{Results}

3.1 Genome-wide Identification of ML domain superfamily and physiochemical properties analysis

291 To identify ML/MD-2 proteins from various fungal species, interpro-id IPRo031672 292 specific for ML/MD-2 proteins was used as a query against the proteome of the target 293 organisms (Supplementary file S1). A varied number of proteins from different organisms 294 were found (Figure 1A). Most of the species had one to three copies of $m l$ gene except $B$. 295 graminis with five and $C$. purpurea with four $m l$ gene copies each. Apart from these two 296 pathogens, the highest numbers of genes (33) were found in $R$. irregularis. Further, these 297 genes were subjected to NCBI CD-search to verify their coding region for the ML domain 298 (Supplementary file S2). The deduced sequences varied between 139 amino acid residues 299 (SCD8PQS8) to a maximum of 1071 amino acid residues (TCL8WPE2). Cysteine content analysis for ML protein showed the highest cysteine content in the Puccinia triticna Pt5643 gene, while CHM2TBXo had the lowest cysteine content (Supplementary file S3, S4, and $\mathrm{S}_{5}$ ).

\subsection{Protein domain, exon/intron structure, and conserved motifs analysis}

The NCBI CD search and Pfam were used to identify the conserved domain present in ML proteins. The results revealed that ML superfamily proteins are enriched with a single ML domain, while some possessed other multiple domains apart from the ML domain (Figure 3). Proteins containing a single ML domain (number) were most abundant, with a 
containing ML domain at N-terminal and TRP-like a dominant domain at C-terminal ends. Proteins exclusively present in oomycetes comprised the third group with two additional domains, the Cathepsin propeptide inhibitor domain at $\mathrm{N}$ - terminal and Peptidase $\mathrm{C} 1 \mathrm{~A}$ papain along with the small-sized ML domain at the extreme $\mathrm{C}$ terminus. The fourth group consisted of two proteins (TCL8WM75, TCL8WPE2) from Thanatephorus cucumer, having three different domains, the SKBP1/BTB/POZ domain and the Nucleophile aminohydrolases and Carbohydrate-Binding Module family (CBM) domain respectively. The exon/intron structural arrangements and their number were similar for species from the same class (Data not shown) Interestingly most of the ascomycetes species had three exons and two introns, except Colletotrichum sublineola (CSAoAo66WWY1) and Saccharomyces cerevisiae (SC02278) which were intronless. The motif size identified by MEME was 4 to 50 amino acids long. Similarity within the motifs across fungal and model host ML proteins showed three major motifs for these proteins (Figure $\mathrm{S} 4$ ). The first group represented the NPC2 domain present in basidiomycetes, while the second motif was represented by PG/PI group proteins, causing the majority of single domain ML (PG/PI) proteins to fall in this group. The third group comprised the same NPC2 domain; however, both the ascomycetes, as well as basidiomycetes proteins, were present in this group.

\subsection{Sequence alignment and characterization of ML superfamily proteins}

Multiple sequence alignment of the identified ML proteins showed very high diversity among and across the fungal species was observed, except in the domain region that was relatively conserved (Figure S1A). All ML proteins generally had four conserved cysteine and basic residues (Lysine and Arginine) in the domain region, which are essentially required for disulfide linkage and making a hydrophobic pocket for ligand binding(Gruber 
333 et al., 2004). Further, the alignment of conserved domain regions revealed the clustering 334 of all of the sequences into four major groups (Figure S1B). Group one included proteins of 335 the PG/PI category present in plants and fungal species only. Group two included proteins 336 from humans, mice, and other animals annotated as GM2-AP of the ML family. The third 337 group consisted of NPC-2 like proteins from drosophila, humans, Clavicipitaceae, Tremellaceae, and rust fungal species. The fourth category was formed by MD-1/MD-2 proteins present only in animals. Except for a few species, most of them were grouped as expected. The identified ML proteins of Clavicipitaceae and Tremellaceae clustered along with the NPC2 and PG/PI proteins, which are from the third and first groups, respectively. However, Puccinia sp ML proteins specifically clustered only with group three NPC2 proteins. These results were quite interesting to observe the unusual presence of NPC2 like 344 domain within the fungal species.

So to investigate the origin of npc2-like genes in P.triticina and other species with NPC2 domain, BLASTp analysis was performed using the NCBI non-redundant (nr) database (1.0e-5, and percentage identity of 30\%) against Pt5643, a $P$. triticina NPC2 like protein. As expected, top hits for the Pucciniaceae family were achieved, followed by hits with species Kockovaella imperatae, Naematelia encephala, and multiple members of Kwoniella, all from family fungi/Tremellaceae, few hits with Culex quinquefasciatus and S7). The results were reconfirmed by performing tBLASTn analysis using Pt5643 as a BLAST query against the NCBI nr database as well as exclusively against fungal taxa. 
355 We found Pucciniaceace and Tremellaceae family members as top hits (Supplementary file 356 S9).

357 Further, BLASTp using Pt5643 as a query against the Insect Base database was performed.

358 Major hits with high e -value found were from C. quinquefasciatus, Anopheles, and 359 Mayetiola destructor all belonging to Diptera (Supplementary file S10 \& S11). Apart from 360 Pt5643 as query, BLAST analysis using previously reported horizontally transferred from 361 Metarhizium and Claviceps npc2- like genes (as a query) with similar parameters 362 demonstrated that NPC2 like proteins are well present in other species like Pochonia 363 chlamydosporia and Ustilaginoidea virens from class Clavicipitaceae (Supplementary 364 file S12). Additionally, Pfam, Interpro, and SMART databases were also searched for ml 365 proteins and annotated with CD-search to explore the possibility of finding any novel 366 NPC2 like proteins; we did not find any other fungal NPC2 like proteins in these databases 367 except for the above-reported species.

\subsection{Phylogenetic studies and selection pressure analysis.}

369 Phylogenetic analysis was performed using the IQ-TREE server (Trifinopoulos et al., 2016). The ML proteins of fungal and oomycetes species identified in this study along with

371 previously known fungal ML proteins from family Clavicipitaceae, as well as from various 372 other eukaryotes (plants, animals, insects, protists (retrieved from Interpro) were 373 combined to compare and infer the existing evolutionary inference. The analysis showed 374 oomycetes ML proteins as a separate clade pointing towards its unrelatedness from fungal 375 species (Fig 2). According to the previous study (Inohara and Nuñez, 2002), fungal ML

376 proteins contain PG/PI domain only. The results showed PG/PI domain proteins cluster in 
377 two separate groups, one containing single PG/PI domain-containing proteins while other

378 TRP domain proteins associated with PG/PI domain. The latter group was found 379 specifically present in ascomycetes ML proteins. In the case of animals, three major 380 subclusters were formed belonging to the class, GM2-AP, MD-1-MD-2, and NPC2, 381 respectively.

382

383

384

385

386

387

388

389

390

391

392

393

394

395

396

397 398 (Table 1).

The ML proteins from Clavicipitaceae ( C. purpurea and Metarhizium robertsii) have already been reported to cluster with animal NPC2 like proteins (Zhao et al., 2014). In the context of this, the results additionally showed ML proteins from other multiple Metarhizium spp to cluster with animals NPC2 proteins. Surprisingly we also found that NPC2 like proteins of other members like $P$. chlamydosporia and U.virens also clustered with animals NPC2 proteins (Fig 2). Apart from Clavicipitaceae (ascomycetes) members, various rust fungal species (basidiomycetes) also clustered with animals NPC2 like proteins, however, forming separate subgroups from both animals NPC2 and Clavicipitaceae suggesting its uniqueness from these species. Though, fungal NPC2 proteins seemed to show more similarity with animals and insects than the rest of the fungal ML proteins (Fig 2). In contrast to so much diversity within the fungal ML proteins, plant PG/PI proteins were present only in one separate cluster supporting the previous reports (Inohara and Nuñez, 2002). Separately, the $\mathrm{Ka} / \mathrm{Ks}$ ratio analysis for orthologous ML genes was also performed. Results demonstrated that most of the ML genes are under purifying selection except two genes from $B$. graminis, one from $P$. striformis, and $R$ irregularis possessed $\mathrm{Ka} / \mathrm{Ks}>1$ and therefore might be under possible positive selection 


\section{distribution of $\mathrm{ml}$ genes}

401 To identify signal peptide and putative subcellular location of identified ML proteins, CBS

402 prediction Servers software was used, and results were summarized in Supplementary

403 Table 1. EffectorP marked 32 proteins out of 84 as putative effector molecules. Overall,

404 these insilico analyses showed that ML proteins are diverse in location and can be found in

405 extracellular secreted form or membrane-bound form to different organelles. The

406 chromosomal location of ML family genes was analyzed by using mapchart software

407 (Figure $\mathrm{S}_{5}$ ).

\subsection{Dimensional structure prediction and molecular docking studies}

To analyze the interaction of $M L$ proteins with the various lipid and sterols, tertiary structures of all ML proteins were examined using various bioinformatics tools. Proteins predictions (Figure 4 and Figure S6 ). For Molecular docking, various sterols and lipids were used, but cholesterol and lanosterol were used for the initial screening of ML

414 proteins. Out of the analyzed species, B. graminis, C. purpurea, $P$. triticina, $P$. sorghi, $P$. 415 striiformis, and R. irregularis showed positive docking using cholesterol, lanosterol, and 416 various ligands (Table 2).

417 Since the ML protein superfamily has been reported to bind with sterols and lipids in 418 various studies (Xu et al., 2007; Zhao et al., 2014), therefore, we focused the study on 419 major sterols like cholesterols, ergosterol, and their homologs, plant-related sterols, 420 Phosphatidylinositol/Phosphatidylglycerol(PI/PG), including other molecules primarily

421 found in cholesterol biosynthesis and various other major sterol synthesis pathways of 
422 fungi. Amongst all the pathogens used in the study, R. irregularis, B. graminis, and $C$. 423 purpurea showed the highest number (33, 5, and 4 respectively) of ML genes. One gene,

424 each form B. graminis and C. purpurea showed positive docking out of all the genes used 425 for docking with various sterol ligands. Further, randomly picked 5 ML genes were used 426 for structure prediction and docking analysis for R.irregularis; All of these genes showed 427 positive interaction with major sterols. One out of the two $P$. triticina ML proteins (the 428 only member from rust fungi (Pt5643) used initially in this study) showed positive docking 429 results (Table 3 ).

430 Further to gain confidence with the results found in P. triticina, other Puccinia species viz. $431 \quad$ P. graminis, $P$. striformis, $P$. sorghi, and $P$. horiana were included in the study. Docking 432 analysis revealed a total of three genes from Puccinia species, one each from $P$. triticna, $P$. 433 striiformis, and $P$. sorghi showing positive interaction (Table 3 ). None of the $m l$ genes 434 from $P$. graminis and $P$. horiana showed any interaction with the sterol ligands. It was 435 interesting to note that all the pathogens showing positive docking interactions were 436 biotrophic in nature. Further, analysis of the amino acid residues involved in interacting 437 with sterols revealed hydrophobic residues to be among the top interacting amino acids 438 followed by polar and charged residues. (Supplementary file S5)

\subsection{Transcript abundance analysis and orthologous gene identification}

The presence and abundance of ML genes in different tissue and infection stages at 441 different time intervals were analyzed using NCBI SRA BLAST search. CDS of selected 442 genes were subjected to SRA BLAST and significant hits (number) with E-value-5, bit 443 score 100 were converted into reads per million $(\mathrm{RPM})($ 
444 https://www.ncbi.nlm.nih.gov/news/11-19-2013-SRA-BLAST). Converted RPM values

445 were plotted as a heatmap using the heat mapper software (Figure8a). The highest hits

446 were represented by $P$. striformis genes followed by genes from B. graminis. The reads

447 abundance of P.stri12 (425.8 RPM) and BGAoAo78N1M3 (805.90 RPM) were highest in

448 the latter stages of infection and showed positive docking interactions. Initial infection

449 stages $(6 \mathrm{~h}, 12 \mathrm{~h}, 24 \mathrm{~h})$ depicted low reads in BGAoAo78N1M3, while a substantial increase

450 was observed at $48 \mathrm{~h}$ and being highest at $5 \mathrm{dpi}$.

451 Orthologous gene identification across the selected pathogens (B. graminis, C. pupurea, $P$.

452 triticina, $M$. oryzae, $R$. irregularis, $C$. sublineolum) showed very few sequences to have

453 orthologue due to high diversity among the species. However, the orthologous gene

454 identification within $R$.irregularis showed numerous genes to be paralogous, probably as

455 a result of duplication events (Supplementary File S13)

3.8 Cloning, In vitro expression, qRT-PCR analysis, and subcellular

457 localization

458 The full length ORF of the gene (Pt5643) with an expected size of 570 bp was amplified 459 using gene-specific primers (Figure $5 \mathrm{~A}$ ) and clone in the p-GEMT vector to confirm the 460 identity of the domain (NPC2 like domain) and overall sequence of the Pt5643 gene. The 461 sequence of Pt5643 gene found after sequencing was again subjected to BLAST in a similar 462 procedure previously followed for finding the similarity among other $n p c 2$ genes of other 463 species. Similar results were found in the case of predicted gene Pt5643 of the P. triticina 464 genome. The SDS-PAGE of the expressed protein showed an expected size of 465 approximately 32kDa (Figure 5B). The qRT-PCR analysis was done for the gene showing 
466 positive docking interaction; however, only Pt5643 showed a single band using RT-PCR

467 primers and was used as a candidate for expression analysis (Figure $5 \mathrm{C}$ )

468 The expression of the Pt5643 gene increased as the infection proceeded up to 5dpi, and 469 after that, started decreasing, suggesting the role of the gene in the course of infection. The 470 subcellular localization of Pt5643 in onion epidermal cells and N. benthamiana suggested

471 it may localize in cytoplasm and nucleus (Figure 5D), suggesting the transport role of

472 Pt5643 as previously reported by other studies for animals and Entamoeba histolytica

473 NPC2 proteins (Bolanos et al., 2016; Frolov et al., 2003; Huang et al., 2007; Zhao et al., 474 2014).

\subsection{Functional complementation assay}

476 The complementation assay of the Pt5643 in the npc2 mutant showed that Pt5643 could

477 partially, however, significantly complement the yeast $n p c 2$ gene. The $n p c 2$ yeast mutants 478 are reported to show enhanced tolerance against acetic acid treatment, whereas the wild 479 type show reduced growth(Kawahata et al., 2006). The spot assay of Pt5643 containing 480 npc2 mutants showed reduced growth in the presence of 100mM acetic acids similar to 481 wild-type yeasts. Whereas npc2 mutants showed significant growth up to(1/1000 482 dilutions) clearly showing the function complementation of yeast npc2 genes by rust 483 Pt5643 gene (Fig. 6). 
486 The transient expression of Pt5643 in N. benthamiana showed Pt5643 could suppress the 487 cell death induced by BAX gene expression. Pt5643, when co-infiltrated with BAX at o hrs, 488 failed to hijack the cell death; however, infiltration after BAX after 12hrs did not show any 489 cell death when infiltrated at the same sport where Pt5643 was infiltrated. Similarly, the 490 infiltration of BAX 24 hrs post infiltration of Pt5643 also masked cell death (Fig.7) Similar 491 to N. benthamiana, the expression of Pt5643 in yeast revealed its cell death perturbing 492 nature. Diluted yeast cells with 0.0001 OD for wild type, npc2 mutant yeasts strains, and 493 Pt5643_npc2 mutants cells when plates on 5 $\mathrm{mM} \mathrm{H} 2 \mathrm{O} 2$ containing plates showed a high 494 number of colonies for the Pt5643_npc2 transformants (approximate 324) than wild type 495 (123) and npc2 mutant (90) (Fig. 8). 


\section{Discussion}

506

507

\subsection{Genome-wide Identification and domain analysis of ML domain superfamily}

The $\mathrm{ml} / \mathrm{md}$-2 gene family is present in lower and higher eukaryotes and has been reported to be involved in lipid binding, sterol trafficking, and providing immunity in humans and insects(Liou et al., 2006; Shi et al., 2012; Shimazu et al., 1999). However, the involvement of ML proteins in sterol trafficking and virulence have been reported for few fungal pathogens (Breakspear et al., 2011; Zhao et al., 2014), none of these proteins have been either identified or characterized in plant fungal pathogens. The present investigation pertains to the identification of $\mathrm{ml}$ gene family members in the 30 different fungal and oomycetes species and further their characterization for host manipulation function. The genome-wide mining identified a total of 84 proteins containing the conserved ML domain, where several proteins harbor other additional domains, including transient receptor potential (TRP) domain, $\mathrm{SKP} 1 / \mathrm{BTB} / \mathrm{POZ}$ domain, cathepsin propeptide inhibitor domain, and peptidase C1A papain domain. The transient receptor potential (TRP) domain is an integral membrane protein domain present in animals and fungi, where they function as ion channels and are essentially required for the function of intracellular organelles such as vacuoles, endoplasmic reticulum, and lysosomes (Cheng et al., 2010; Puertollano and Kiselyov, 2009). In the present study, 12 out of the 84 identified ML proteins were found to contain the TRP domain and were shown to retain their membrane-bound property (Table 2). In yeast, the TRP protein-encoding gene has been reported to be involved in intracellular calcium ion transport and signaling through the vacuolar membrane (Denis and Cyert, 2002; Palmer et al., 2001). A similar finding has been 
528

529

530

531

532

533

534

535

536

537

538

539

540

541

542

543

544

545

546

547

548

549

550

reported for Gibberella zeae (ascomycetes) fungal TRP protein, TRPGz, where TRPY1 knock-out yeast cell expressing fungal TRPGz showing activation under hyperosmolarity, temperature changes, elevated cytosolic $\mathrm{Ca}^{2+}$ ion concentration, membrane potential after $\mathrm{H}_{2} \mathrm{O}_{2}$ treatment (Ihara et al., 2013). These previous studies suggest that the TRP domaincontaining ML proteins identified in the present study might play a similar role in the environmental stimuli signaling in case plant pathogenic fungi of the class ascomycetes.

The presence of cathepsin propeptide inhibitor domain and peptidase $\mathrm{C} 1 \mathrm{~A}$ papain domain along with ML domain has been found exclusively in oomycetes ML proteins, which suggests functional divergence of these proteins from fungal ML proteins. Intriguingly, in contrast to the investigated fungal species, only members of Pucciniaceae have been found to contain NPC2 like domain-containing proteins pointing towards its divergence from the rest of the fungi. Although members of Clavicipitaceae have been previously reported to have NCP2 like proteins with lipid-binding function (Zhao et al., 2014), no comparative analysis has been performed across the fungal species.

The identification of ML proteins showed a limited number of ML proteins in fungal species; however, a huge expansion of the ML protein family was observed in mycorrhizal fungus Rhizophagus irregularis (Figure 1 B) an obligate biotroph. The fungi and plants are known to contain PG/PI proteins, which are involved in binding with phosphatidylglycerol (PG) and phosphatidylinositol (PI), where PG and PI are important messenger molecules involved in lipid-based signaling (Inohara and Nuñez, 2002). The presence of a high number of $R$. irregularis PG/PI subfamily members suggests that they might be involved in hijacking or interfering with plant lipid-based signaling pathways for successful establishment inside the plant. Previous studies have reported the high expression of the 
551 majority of PG/PI genes in R.irregularis during infection progression and have also been

552 defined as effectors in $R$. clarus(Chen et al., 2018; Toro and Brachmann, 2016; Zeng et al.,

553 2018). Additionally, multiple secretory nonspecific lipid transfer proteins have been

554 identified, responsible for the transfer of lipids from host to mycorhizal fungi and further

555 recycling (Bago et al., 2002; Jiang et al., 2017; Keymer et al., 2017; Luginbuehl et al., 2017;

556 Zeng et al., 2018). Recent studies have also reported the presence of palmitic acid in the $R$.

557 irregularis hyphae, irrespective of the complete absence of lipid biosynthesis pathway 558 genes, indicating the accumulation of palmitic acid through transport from the host 559 (Wewer et al., 2014).

\subsection{Phylogenetic analysis of ML domain proteins}

561 The initial studies on the identification of ML gene family in all eukaryotes, including plants, animals, and fungi, identifies only PG/PI type ML proteins in plants and fungi (Inohara and Nuñez, 2002). The absence of NPC2 like proteins in all the majority of fungal species suggests that $n p c 2$ like genes have never been a part of fungal species in due course insect pathogenic fungi (Metarhizium robertsiii, Metarhizium acridum, and Claviceps aminal NPC2 proteins. Zhao et al., found these genes to be horizontally transferred from the host insect of Clavicipitaceae. However, no studies was previously performed since then to identify ML domain (npc2 like genes) genes in plant fungal pathogens or any other

571 fungal species. The phylogetic analysis of 68 different eukaryotes (Fungi-22, Plants-6, 572 oomycetes-5, protists-28, animals-5) clearly showed that most of fungi contain PG/PI 573 members and clutster separately from animal NPC2, however members from the 
574 Pucciniaceae ( 6 species) and previously reported HGT candidate ( C. pupurea , 575 Cpurp_M1W4K5) from family Clavicipitaceae clearly clustered with animal ML proteins 576 especially with insect NPC2 members suggesting their similarity to animal NPC2 than 577 fungal ML proteins (PG/PI).

The similarity of Pucciniaceae ML members, with NPC2 like proteins of insects and animals in the phylogenetic analysis, also supported the findings of Zhao et al., 2014 (Fig.2). Besides, the molecular docking studies also reveal the putative cholesterol binding activity of Puccinia NPC2 proteins, a characteristic of animals and insects NPC2 proteins, thus, indicates their functional conservation across the kingdoms.

An insight into the evolutionary history before diversification revealed that both animals and fungi evolved from the common ancestor "Opisthokonts" and were expected to share the common gene pool. However, the phylogenetic analysis showed the complete absence of the NPC2 class of ML proteins in all the 22 major plant pathogens studied (17 fungal pathogens and 5 oomycetes ). From the evolutionary point of view, there are very fewer chances that selection pressure simultaneously led to the loss of genes in multiple organisms. So, it could be possible that identified fungal npc2 like genes have been evolved independently from plant/fungi-specific $P G / P I$ genes in these fungal species. However, the chances of $n p c 2$ evolution in Puccinia spp. from $p g / p i$ is highly unlikely since none of the Puccinia spp. (six spp.) were found to contain $p g / p i$ genes (even with the BLAST hit score). Therefore, one of the possible mechanisms of acquisition of npc2 by Puccinia spp. could be horizontal gene transfer (HGT) from animals, insects, or other fungi containing npc2 genes. Since Puccinia spp. and Claviceps spp. both infect the wheat plant; therefore, there may be chances of npc2 like genes getting transferred to Puccinia spp. from 
597 Claviceps spp. However, the npc2 genes of Clavicipitaceae members, including Claviceps, 598 clustered separately in the phylogeny away from the Puccinia spp., (Figure 2, Figure S2 599 and S3) suggesting its independent origin, rather than from Claviceps spp.

600 Since the members of Clavicipitaceae are entomopathogenic fungi and are known to 601 interact with insects, a previous report for Clavicipitaceae members, C. pupurea, and 602 Metarhizium spp. suggested the origin of npc2 like genes in these fungi through HGT from 603 host insects (Zhao et al., 2014). Unlike Clavicipitaceae, the rust fungal pathogens 604 (Puccinia spp.) are not known to feed on the insects; however, there are examples where 605 rust fungi interact with insects in the environment. The interaction of rust fungi and 606 insects may occur through either a vector-host system for spore transmission (Stephanie et al., 2001; Wandeler and Bacher, 2006) or fungal spore infestation. The rust spore 608 infestation by insects of the Diptera class is a widespread and frequent phenomenon 609 (Henk et al., 2011), suggesting that Puccinia spp. npc2 genes might also be originated from 610 insects through the HGT event, similar to that of Clavicipitaceae members. The hypothesis 611 that Puccinia spp. npc2 genes could be an HGT event from insects appears to be more 612 valid as Drosophila NPC2 proteins were clustered together with NPC2 proteins of 613 Puccinia spp ( Fig. 2). Also, the significant BLASTp score with more than $30 \%$ similarity of 614 these clustered NPC2 proteins, and similar 6o-64\% GC content of encoding genes (45-50\% 615 GC content for the rest of the ML gene family members), suggests that members of the 616 Diptera family could be the possible donor insect species for Puccinia npc2 genes.

617 Moreover, the npc2 genes have also been identified in few other fungal species of genus viz 618 Kwoniella spp., P.chlamydosporia, and Ustilaginoidea virens, where all of these fungal 619 species are known to interact either with insects or nematodes in the environment. 
620

621

622

623

624

625

626

627

628

629

630

631

632

633

634

635

636

637

638

639

640

Therefore, the HGT from insects could be the only possible mechanism to explain the origin of $n p c 2$ genes in these fungal species. However, experimental validation is required to negate neo-functionalization and to provide supporting evidence for HGT events.

\subsection{D-Structure prediction, molecular docking, and selection pressure} analysis

The molecular docking revealed that three NPC2 like proteins of Puccinia spp (Pt5643, P_striiiformis_12, Psorghi_66), and one each from $C$.pupurea (CpM1WA48) and $B$. graminis (BGAoAo78N1M3) exhibit binding with cholesterol well as with various sterols like ergosterol, lanosterol, fungisterol, etc ( Table 2 ). Previously it was reported that NPC2 proteins have the capacity to bind with various sterols because of basic amino acid clusters inside the deep hydrophobic pocket (Liou et al., 2006). The 3D structure analysis of NPC2 like proteins identified in the present study also revealed the presence of basic amino acids (valine, isoleucine, alanine, and phenylalanine) containing deep hydrophobic pockets able to bind with diverse sterols with a high docking score. The superimposition analysis and low Root-Mean-Square Deviation RMSD score (1-2) of these proteins during superimposition with bovine NPC-2 protein crystal structure (data not shown) suggest a functional similarity of these proteins with animal NPC-2 proteins. Besides, all the five proteins also showed significant docking scores with isopentenyl pyrophosphate (IPP), geranyl pyrophosphate (GPP), and farnesyl pyrophosphate (FPP) (Table 3), a precursor of various phytoalexins produced against infection of fungi and various pathogens by the plants (Chávez-Moctezuma and Lozoya-Gloria, 1996; Walters et al., 2008). 
641 All the three fungal pathogens (Puccinia spp, C.purpurea, and B.graminis) are biotrophic 642 and dependent on the host for its survival and propagation, so it could be expected that 643 fungal NPC-2 like proteins have retained the conserved functions of lipid trafficking for 644 fungal survival or engaging the sterols or lipids responsible for signaling that could 645 otherwise reveal the presence of pathogen inside the host cell. Therefore, it could be suggested that NPC2 like proteins are involved in processes against the plant defense 647 mechanism. In addition, the value of $\mathrm{Ka} / \mathrm{Ks}$ for a few of the fungal $n p c-2$ like genes was 648 found to be less than one. which suggests purifying selection pressure and further 649 corroborate the functional conservation of NPC2 like genes in fungus.

\subsection{Functional complementation assay}

651 In the case of npc2 genes, the studies have reported that wild-type yeast show reduced 652 growth in acetic acid, while mutant npc2 strains are resistant to the treatment(Kawahata et 653 al., 2006). The functional complementation of one of the P.triticina gene Pt5643 with 654 yeast npc2 (ML homolog) homolog in the presence of acetic acid (100mM) shows that 655 Pt5643 partially however significantly complements the function npc2 mutant showing 656 phenotype similar to wild type yeast, further suggesting the Pt5643 to play a role similar to 657 yeasts NPC2 (sterol trafficking). Though the Pt5643 gene did not complement the function 658 of later completely, it may play a relatively diverged function in Puccinia spp.

$659 \quad 4.5 \quad P t 5643$ transient expression under BAX and $\mathrm{H2O2}$ induced cell death in 660 N. benthamiana and S. cerevisiae

661 The $\mathrm{BAX}$ and $\mathrm{H}_{2} \mathrm{O}_{2}$ induced program cell death (PCD) hijacking capacity of Pt5643 gene 662 after expressing in $N$. benthamiana and $S$. cerevisiae further suggested its putative 
663 function in P.triticina as a potential effector. In the case of biotrophic pathogens, hijacking 664 of the host induced cell death by interfering with host immune signaling is one of the 665 effector's primary functions. Moreover, cholesterol is also known to play a crucial role in 666 BAX-mediated program cell death (PCD)(Lucken-Ardjomande et al., 2008). The 667 cholesterol accumulation in the cell regulates PCD. At low concentrations, cholesterol 668 promotes the BAX-induced cell death, while high concentration inhibits by changing the 669 membrane property(Li et al., 2020; Mignard et al., 2014). The molecular docking of 670 Pt5643 has shown it to bind cholesterol, and further subcellular localization also showed

671 them to be cytoplasm localized. Therefore, the cell death hijacking function of the Pt5643 672 could be due to its cholesterol trafficking and further accumulation in the cell.

673 The overall results in the study showed that NPC2 like proteins in fungal pathogens might 674 bind to various sterols present in the sterol synthesis pathway, perform trafficking 675 functions, and are probably originated from animal/insect species. Insects are in 676 continuous interaction with fungal pathogens since spores of biotrophic fungal pathogens 677 could be an easy source of nutrition for mycophagous insects. Therefore, the presence of 678 insect-related genes in the Puccinia sp, Claviceps sp, and Tremellales sp might result from 679 the interaction between ancestors of insects and these pathogens and for possible HGT 680 events. Moreover, its presence in a large number of Pucciniaceae members suggests its 681 important role as a conserved effector that hijacks the basic biological process in PCD. 


\section{Conclusions}

687 This study sheds light on the status of the $m l$ gene family in plant pathogenic fungi, and its 688 possible role in plant-pathogen interactions. The ML domain is also associated with 689 various other domains and putatively involved in diverse functions. The results also 690 showed that NPC2 like proteins of Pucciniaceae, Claviceptaecace, and Tremellaceae have 691 possible insect origin and are transferred to these pathogens through the HGT mechanism. 692 Most of the $m l$ gene family members in fungi are going through purifying selection except 693 a few B.graminis, P.striiformis, and R.irregularis $m l$ genes with strong positive selection 694 pressure. The npc2- like genes are already reported to perform sterol transport in humans 695 and other animals, including insects, in association with the npc1 gene across the 696 membrane. The acquisition of npc2 like effector genes and expansion in biotrophic

697 pathogens like rust, Claviceps, $R$. irregularis could be associated with their obligate 698 biotrophic nature, as these pathogens hijack the host signaling system to remain 699 undetected and require raw materials to sustain inside the host. 


\section{Acknowledgments}

TRS is thankful to the Department of Science and Technology, Govt. of India, for JC Bose

National Fellowship. RJ is thankful ${ }^{i}$ to the University Grants Commission (UGC), New

Delhi for providing Junior Research Fellowship (JRF).

\section{Conflict of Interests}

712 The authors declare no conflict of interests

\section{CRediT authorship contribution statement}

714 Rajdeep Jaswal: Conceptualization, Methodology, Software, Analysis, Data curation, 715 Experiments, Writing - original draft. Himanshu Dubey: Formal analysis. Kanti 716 Kiran: Formal analysis. Hukam Rawal: Formal analysis. Sivasubramanian 717 Rajarammohan: Formal analysis. Humira Sonah: Formal analysis. Rupesh 718 Deshmukh- Formal analysis. Pramod Prasad: resource. Subhash C Bhardwaj: 719 resource. Naveen Gupta- Supervision. Tilak Raj Sharma: Conceptualization, 720 Methodology, Supervision, Funding acquisition, writing- review, and editing. 


\section{References}

Altschul, S.F., Gish, W., Miller, W., Myers, E.W., Lipman, D.J., 1990. Basic local alignment search tool. J. Mol. Biol. 215, 403-410.

Ao, J., Ling, E., Rao, X., Yu, X.-Q., 2008. A novel ML protein from Manduca sexta may function as a key accessory protein for lipopolysaccharide signaling. Mol. Immunol. 45, 2772-2781.

Bago, B., Zipfel, W., Williams, R.M., Jun, J., Arreola, R., Lammers, P.J., Pfeffer, P.E., Shachar-Hill, Y., 2002. Translocation and utilization of fungal storage lipid in the arbuscular mycorrhizal symbiosis. Plant Physiol. 128, 108-124.

Bikadi, Z., Hazai, E., 2009. Application of the PM6 semi-empirical method to modeling proteins enhances docking accuracy of AutoDock. J. Cheminform. 1, 15.

Bolanos, J., Betanzos, A., Javier-Reyna, R., Garcia-Rivera, G., Huerta, M., Pais-Morales, J., Gonzalez-Robles, A., Rodriguez, M.A., Schnoor, M., Orozco, E., 2016. EhNPC1 and EhNPC2 proteins participate in trafficking of exogenous cholesterol in Entamoeba histolytica trophozoites: relevance for phagocytosis. PLoS Pathog. 12, e1006089.

Breakspear, A., Pasquali, M., Broz, K., Dong, Y., Kistler, H.C., 2011. Npc1 is involved in sterol trafficking in the filamentous fungus Fusarium graminearum. Fungal Genet. Biol. 48, 725-730.

Chávez-Moctezuma, M.P., Lozoya-Gloria, E., 1996. Biosynthesis of the sesquiterpenic phytoalexin capsidiol in elicited root cultures of chili pepper (Capsicum annuum). Plant Cell Rep. 15, 360-366. 
748

749

750

751

752

753

754

755

756

757

758

759

760

761

762

763

764

765

766

767

768

Chen, E.C.H., Morin, E., Beaudet, D., Noel, J., Yildirir, G., Ndikumana, S., Charron, P., StOnge, C., Giorgi, J., Krüger, M., 2018. High intraspecific genome diversity in the model arbuscular mycorrhizal symbiont Rhizophagus irregularis. New Phytol. 220, $1161-1171$.

Cheng, X., Shen, D., Samie, M., Xu, H., 2010. Mucolipins: Intracellular TRPML1-3 channels. FEBS Lett. 584, 2013-2021.

Coelho, M.A., Gonçalves, C., Sampaio, J.P., Gonçalves, P., 2013. Extensive intra-kingdom horizontal gene transfer converging on a fungal fructose transporter gene. PLoS Genet. 9, e1003587.

Denis, V., Cyert, M.S., 2002. Internal Ca2+ release in yeast is triggered by hypertonic shock and mediated by a TRP channel homologue. J Cell Biol 156, 29-34.

Dong, Y., Aguilar, R., Xi, Z., Warr, E., Mongin, E., Dimopoulos, G., 2006. Anopheles gambiae immune responses to human and rodent Plasmodium parasite species. PLoS Pathog 2, e52.

Fitzpatrick, D.A., 2012. Horizontal gene transfer in fungi. FEMS Microbiol. Lett. 329, 1-8.

Frolov, A., Zielinski, S.E., Crowley, J.R., Dudley-Rucker, N., Schaffer, J.E., Ory, D.S., 2003. NPC1 and NPC2 regulate cellular cholesterol homeostasis through generation of LDLderived oxysterols. J. Biol. Chem.

Gruber, A., Manček, M., Wagner, H., Kirschning, C.J., Jerala, R., 2004. Structural model of MD-2 and functional role of its basic amino acid clusters involved in cellular lipopolysaccharide recognition. J. Biol. Chem. 279, 28475-28482. 
Halgren, T.A., 1996. Merck molecular force field. I. Basis, form, scope, parameterization, and performance of MMFF94. J. Comput. Chem. 17, 490-519.

Henk, D.A., Farr, D.F., Aime, M.C., 2011. Mycodiplosis (Diptera) infestation of rust fungi is frequent, wide spread and possibly host specific. Fungal Ecol. 4, 284-289.

Huang, X., Warren, J.T., Buchanan, J., Gilbert, L.I., Scott, M.P., 2007. Drosophila Niemann-Pick type C-2 genes control sterol homeostasis and steroid biosynthesis: a model of human neurodegenerative disease. Development 134, 3733-3742.

Ihara, M., Hamamoto, S., Miyanoiri, Y., Takeda, M., Kainosho, M., Yabe, I., Uozumi, N., Yamashita, A., 2013. Molecular bases of multimodal regulation of a fungal transient receptor potential (TRP) channel. J. Biol. Chem. 288, 15303-15317.

Inohara, N., Nuñez, G., 2002. ML-a conserved domain involved in innate immunity and lipid metabolism. Trends Biochem. Sci. 27, 219-221.

Jaswal, R., Dubey, H., Kiran, K., Singh, P.K., Rawal, H.C., Bhardwaj, S.C., Prasad, P., Gupta, N., Sharma, T.R., 2019. Comparative secretome analysis of Indian wheat leaf rust pathogen Puccinia triticina. Indian J. Agric. Sci. 89, 1688-1692.

Jaswal, R., Kiran, K., Rajarammohan, S., Dubey, H., Singh, P.K., Sharma, Y., Deshmukh, R., Sonah, H., Gupta, N., Sharma, T.R., 2020a. Effector Biology of Biotrophic Plant Fungal Pathogens: Current Advances and Future Prospective. Microbiol. Res. 126567.

Jaswal, R., Rajarammohan, S., Dubey, H., Sharma, T.R., 2020b. Smut fungi as a stratagem to characterize rust effectors: opportunities and challenges. World J. Microbiol. Biotechnol. 36, 1-10. 
790

791

792

793

794

795

796

797

798

799

800

801

802

803

804

805

806

807

808

809

810

811

Jiang, Y., Wang, W., Xie, Q., Liu, N., Liu, L., Wang, D., Zhang, X., Yang, C., Chen, X., Tang, D., 2017. Plants transfer lipids to sustain colonization by mutualistic mycorrhizal and parasitic fungi. Science (80-. ). eaam9970.

Johannessen, B.R., Skov, L.K., Kastrup, J.S., Kristensen, O., Bolwig, C., Larsen, J.N., Spangfort, M., Lund, K., Gajhede, M., 2005. Structure of the house dust mite allergen Der f 2: Implications for function and molecular basis of IgE cross-reactivity. FEBS Lett. 579, 1208-1212.

Jupatanakul, N., Sim, S., Dimopoulos, G., 2014. Aedes aegypti ML and Niemann-Pick type C family members are agonists of dengue virus infection. Dev. Comp. Immunol. 43, 19.

Juretic, N., Hoen, D.R., Huynh, M.L., Harrison, P.M., Bureau, T.E., 2005. The evolutionary fate of MULE-mediated duplications of host gene fragments in rice. Genome Res. 15, 1292-1297.

Kawahata, M., Masaki, K., Fujii, T., Iefuji, H., 2006. Yeast genes involved in response to lactic acid and acetic acid: acidic conditions caused by the organic acids in Saccharomyces cerevisiae cultures induce expression of intracellular metal metabolism genes regulated by Aft1p. FEMS Yeast Res. 6, 924-936.

Keymer, A., Pimprikar, P., Wewer, V., Huber, C., Brands, M., Bucerius, S.L., Delaux, P.-M., Klingl, V., von Roepenack-Lahaye, E., Wang, T.L., 2017. Lipid transfer from plants to arbuscular mycorrhiza fungi. Elife 6, e29107.

Kim, H.M., Park, B.S., Kim, J.-I., Kim, S.E., Lee, J., Oh, S.C., Enkhbayar, P., Matsushima, N., Lee, H., Yoo, O.J., 2007. Crystal structure of the TLR4-MD-2 complex with bound 
endotoxin antagonist Eritoran. Cell 130, 906-917.

813

814

815

816

817

818

819

820

821

822

823

824

825

826

827

828

829

830

831

832

Kiran, K., Rawal, H.C., Dubey, H., Jaswal, R., Bhardwaj, S.C., Prasad, P., Pal, D., Devanna, B.N., Sharma, T.R., 2017. Dissection of genomic features and variations of three pathotypes of Puccinia strïformis through whole genome sequencing. Sci. Rep. 7.

Kiran, K., Rawal, H.C., Dubey, H., Jaswal, R., Devanna, B.N., Gupta, D.K., Bhardwaj, S.C., Prasad, P., Pal, D., Chhuneja, P., 2016. Draft genome of the wheat rust pathogen (Puccinia triticina) unravels genome-wide structural variations during evolution. Genome Biol. Evol. 8, 2702-2721.

Kumar, S., Stecher, G., Tamura, K., 2016. MEGA7: Molecular Evolutionary Genetics Analysis version 7.0 for bigger datasets. Mol. Biol. Evol. msw054.

Li, K., Deng, Y., Deng, G., Chen, P., Wang, Y., Wu, H., Ji, Z., Yao, Z., Zhang, X., Yu, B., 2020. High cholesterol induces apoptosis and autophagy through the ROS-activated AKT/FOXO1 pathway in tendon-derived stem cells. Stem Cell Res. Ther. 11, 1-16.

Li, W.-H., Gojobori, T., Nei, M., 1981. Pseudogenes as a paradigm of neutral evolution. Nature 292, 237-239.

Liao, J.-X., Yin, Z.-X., Huang, X.-D., Weng, S.-P., Yu, X.-Q., He, J.-G., 2011. Cloning and characterization of a shrimp ML superfamily protein. Fish Shellfish Immunol. 30, 713-719.

Librado, P., Rozas, J., 2009. DnaSP v5: a software for comprehensive analysis of DNA polymorphism data. Bioinformatics 25, 1451-1452.

Liou, H.-L., Dixit, S.S., Xu, S., Tint, G.S., Stock, A.M., Lobel, P., 2006. NPC2, the protein 
deficient in Niemann-Pick C2 disease, consists of multiple glycoforms that bind a variety of sterols. J. Biol. Chem. 281, 36710-36723.

835

836

837

838

839

840

841

842

843

844

845

846

847

848

849

850

851

852

853

Livak, K.J., Schmittgen, T.D., 2001. Analysis of relative gene expression data using realtime quantitative PCR and the 2- $\Delta \Delta \mathrm{CT}$ method. methods $25,402-408$.

Lucken-Ardjomande, S., Montessuit, S., Martinou, J.-C., 20o8. Bax activation and stressinduced apoptosis delayed by the accumulation of cholesterol in mitochondrial membranes. Cell Death Differ. 15, 484-493.

Luginbuehl, L.H., Menard, G.N., Kurup, S., Van Erp, H., Radhakrishnan, G. V, Breakspear, A., Oldroyd, G.E.D., Eastmond, P.J., 2017. Fatty acids in arbuscular mycorrhizal fungi are synthesized by the host plant. Science (80-. ). eaanoo81.

Mignard, V., Lalier, L., Paris, F., Vallette, F.M., 2014. Bioactive lipids and the control of Bax pro-apoptotic activity. Cell Death Dis. 5, e1266-e1266.

Palmer, C.P., Zhou, X.-L., Lin, J., Loukin, S.H., Kung, C., Saimi, Y., 2001. A TRP homolog in Saccharomyces cerevisiae forms an intracellular Ca2+-permeable channel in the yeast vacuolar membrane. Proc. Natl. Acad. Sci. 98, 7801-7805.

Puertollano, R., Kiselyov, K., 2009. TRPMLs: in sickness and in health. Am. J. Physiol. Physiol. 296, F1245-F1254.

Qiu, H., Cai, G., Luo, J., Bhattacharya, D., Zhang, N., 2016. Extensive horizontal gene transfers between plant pathogenic fungi. BMC Biol. 14, 41.

Shi, X.-Z., Zhong, X., Yu, X.-Q., 2012. Drosophila melanogaster NPC2 proteins bind bacterial cell wall components and may function in immune signal pathways. Insect 
Biochem. Mol. Biol. 42, 545-556.

855

856

857

858

859

860

861

862

863

864

865

866

867

868

869

870

871

872

873

874

Shimazu, R., Akashi, S., Ogata, H., Nagai, Y., Fukudome, K., Miyake, K., Kimoto, M., 1999. MD-2, a molecule that confers lipopolysaccharide responsiveness on Toll-like receptor 4. J. Exp. Med. 189, 1777-1782.

Slot, J.C., Rokas, A., 2011. Horizontal transfer of a large and highly toxic secondary metabolic gene cluster between fungi. Curr. Biol. 21, 134-139.

Stephanie, K., Andreas, K., Teja, T., 2001. Interactions between the rust fungus Puccinia punctiformis and ectophagous and endophagous insects on creeping thistle. J. Appl. Ecol. 38, 548-556.

Toro, K.S., Brachmann, A., 2016. The effector candidate repertoire of the arbuscular mycorrhizal fungus Rhizophagus clarus. BMC Genomics 17, 101.

Trifinopoulos, J., Nguyen, L.-T., von Haeseler, A., Minh, B.Q., 2016. W-IQ-TREE: a fast online phylogenetic tool for maximum likelihood analysis. Nucleic Acids Res. 44, W232-W235.

Vizzini, A., Bonura, A., Longo, V., Sanfratello, M.A., Parrinello, D., Cammarata, M., Colombo, P., 2015. Isolation of a novel LPS-induced component of the ML superfamily in Ciona intestinalis. Dev. Comp. Immunol. 53, 70-78.

Walters, D., Newton, A.C., Lyon, G., 2008. Induced resistance for plant defence: a sustainable approach to crop protection. John Wiley \& Sons.

Wandeler, H., Bacher, S., 2006. Insect-transmitted urediniospores of the rust Puccinia punctiformis cause systemic infections in established Cirsium arvense plants. 
875

876

877

878

879

880

881

882

883

884

885

886

887

888

889

890

891

892

893

894

Phytopathology 96, 813-818.

Wewer, V., Brands, M., Dörmann, P., 2014. Fatty acid synthesis and lipid metabolism in the obligate biotrophic fungus Rhizophagus irregularis during mycorrhization of $\mathrm{L}$ otus japonicus. Plant J. 79, 398-412.

Xu, D., Zhang, Y., 2011. Improving the physical realism and structural accuracy of protein models by a two-step atomic-level energy minimization. Biophys. J. 101, 2525-2534.

Xu, S., Benoff, B., Liou, H.-L., Lobel, P., Stock, A.M., 2007. Structural basis of sterol binding by NPC2, a lysosomal protein deficient in Niemann-Pick type C2 disease. J. Biol. Chem. 282, 23525-23531.

Yang, J., Yan, R., Roy, A., Xu, D., Poisson, J., Zhang, Y., 2015. The I-TASSER Suite: protein structure and function prediction. Nat. Methods 12, 7-8.

Yin, Z., Zhu, B., Feng, H., Huang, L., 2016. Horizontal gene transfer drives adaptive colonization of apple trees by the fungal pathogen Valsa mali. Sci. Rep. 6, 33129.

Zeng, T., Holmer, R., Hontelez, J., te Lintel-Hekkert, B., Marufu, L., de Zeeuw, T., Wu, F., Schijlen, E., Bisseling, T., Limpens, E., 2018. Host-and stage-dependent secretome of the arbuscular mycorrhizal fungus Rhizophagus irregularis. Plant J. 94, 411-425.

Zhao, H., Xu, C., Lu, H.-L., Chen, X., Leger, R.J.S., Fang, W., 2014. Host-to-pathogen gene transfer facilitated infection of insects by a pathogenic fungus. PLoS Pathog 10, e1004009. 
Fig. 1. Genome-wide identification of $\mathrm{ml}$ gene family in various fungal species. (A) ML domain proteins identified in major fungal and oomycetes species. Among biotrophs, Blumeria garminis and Claviceps purpurea had the highest ML proteins 5 and 4, respectively, while other species (hemibiotrophs, necrotrophs, saprotrophs and oomycetes mostly enode1-3 copies. (B) Comparison of identified ML proteins of various fungal species with mycorrhizal fungus $R$. irregularis (RI)., the highest number of $\mathrm{ML}$ domain proteins (33) were found in RI, suggesting an expansion of protein domain family.

Fig. 2. Phylogenetic distribution of ML domain protein family. Phylogenetic relationship of ML proteins in plants, animals, insects, protists and fungi: the proteins clustered in four subclasses: ML Animals (MD-1,GM2A, NPC2), plant, green algae and fungi (PG/PI), Fungi HGT events (NPC2 (Pucciniaceae and Clavicipitaceae ). For tree construction, maximum likelihood method with 1000 bootstrap runs was used). The tree was generated using IQ-TREE software.

Fig. 3. Domain organization of ML protein family (A) Domain organization of ML and associated domain in fungal and oomycetes proteins: the size of the domains used only for representation and does not represent the actual size. Most fungal species contain a single ML domain; however, few members from ascomycetes have double domain annotated as TRP (Transient Receptor Potential), while oomycetes ML members contain Cathepsin Propeptide Inhibitor domain (CPID) and PCPC-Peptidase C1A papain (PCPC). (B) Domain organization of ML proteins for host and model species: most of the hosts (animal and plant ML proteins) have a single ML domain, unlike fungal and oomycetes ML proteins.

Fig. 4. The 3D Structure prediction and molecular docking analysis of ML domain proteins. Few structures were used here for representing the structure of ML proteins. (A) NPC2 like protein of P.triticina. Figure (B) PG/PI protein of M.oryzae. (C) 
PG/PI TRP protein of S.reilianum. D) ML protein of P.ramorum. (E) \& (F) Molecular Docking of Pt5643 (NPC2 like proteins) of P.triticina with cholesterol. The cholesterol showed the highest docking score with Pt_5643. Ribbon and surface forms of $3 \mathrm{D}$ structures were used to highlight the interactions. 3D structure of all proteins is available in Figure $\mathrm{S}_{5}$ (Supplementary data).

Fig. 5. Characterization of npc2 like gene of $P$. triticina (Pt5643) P. triticina infected Wheat leaf samples were used for RNA extraction and cDNA synthesis. (A) PCR amplification of Pt5643 gene with a band of 570bp from the infected leaf sample cDNA. (B) Invitro expression analysis of Pt5643 in 12\%SDS-PAGE gel giving a band of 32kDa in pET29a vector. (C) Expression analysis of Pt5643 in the infection cycle. (D) The subcellular localization of Pt5643 in onion epidermal cells ( upper two panel) using the particle bombardment method and and N.benthamiana (lower panel) using agroinfiltration shows its location in the nucleus and cytoplasm.

Fig. 6. Complementation assay of the Pt5643 gene in the npc2 mutant of $S$. cerevisiae. The wild-type yeasts are unable to grow on $100 \mathrm{mM}$ acetic acid, while npc2 mutants can grow. The npc2 mutant containing Pt5643 shows reduced growths when compared to the npc2 mutant alone, show

Fig. 7. BAX induced cell death suppression assay of Pt5643 gene in $N$. benthamiana using agroinfiltration method. (A). Infiltration of tobacco leaves and phenotype analysis. (B). Leaf without chlorophyll treated with alcohol and acetic acid mixture (70\% ethanol + 10\% acetic acid). (1.pgwb408- empty vector, 2.Pt5643_pgwb408, 3.BAX+ pGWB408, 4.BAX_pgwb408, 5.Bax at oh, 6.BAX + Pt5643 at o h, 7.Buffer ; after 12 h BAX, 8.Pt5643; after 12 h BAX, 9. Buffer; after 24 h BAX, 10. Pt5643; after 24h Bax). 
Fig. 8. $\mathrm{H}_{2} \mathrm{O}_{2}$ induced cell death suppression assay of Pt5643 gene in $\mathrm{S}$. cerevisiae. Cells with 0.0001 each for wild type, npc2 mutant and Pt5643 containing npc2 cells were plated on 5mM $\mathrm{H}_{2} \mathrm{O} 2$ containing YPD plates. Higher numbers of colonies approximately (324)were rescued for Pt5643 containing npc2 cells than wild type (123) and npc2 mutant alone (90).

Fig. 1. 
bioRxiv preprint doi: https://doi.org/10.1101/2021.08.18.456830; this version posted August 18, 2021. The copyright holder for this preprint (which was not certified by peer review) is the author/funder. All rights reserved. No reuse allowed without permission.

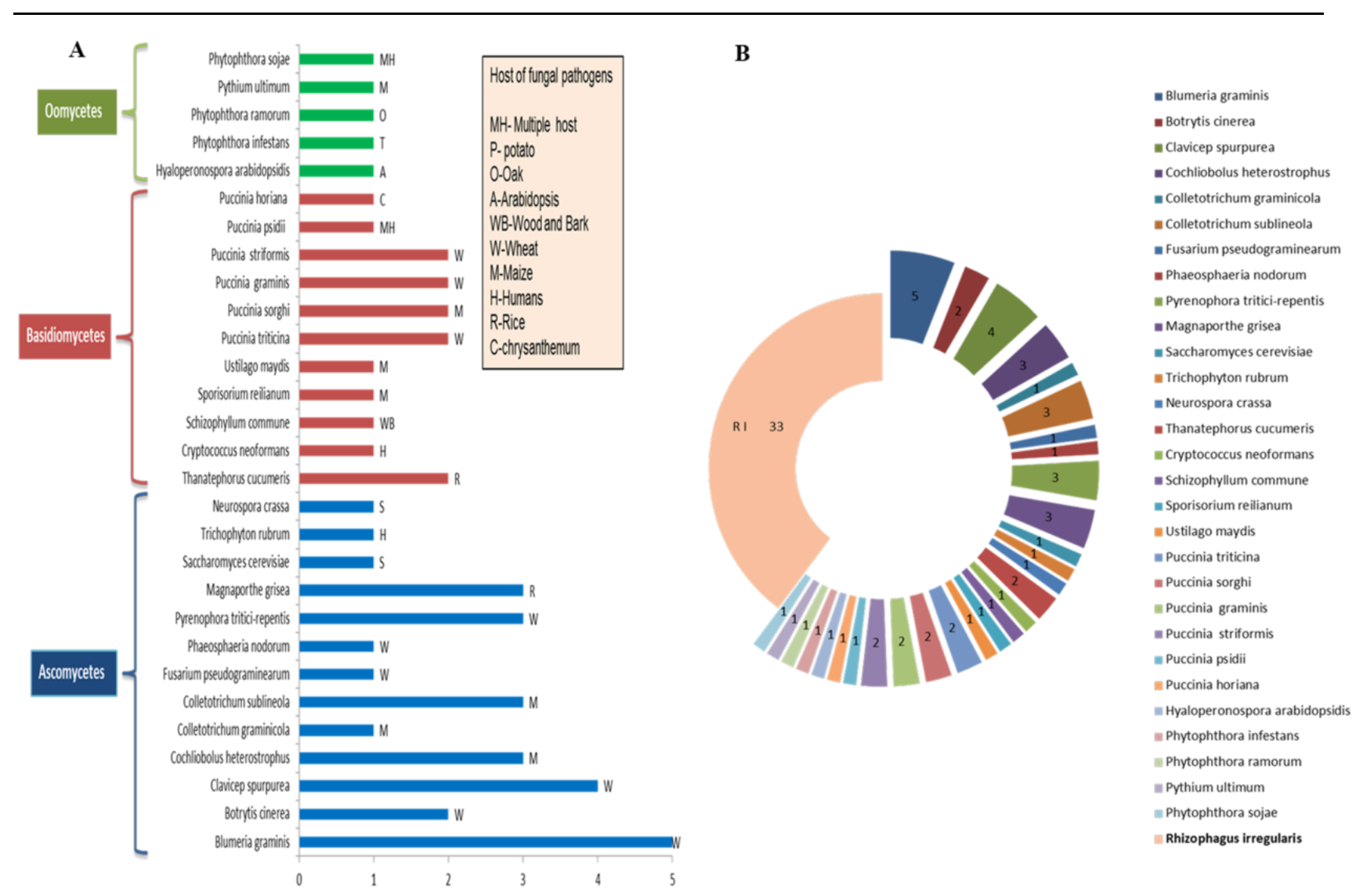

Fig. 2. 


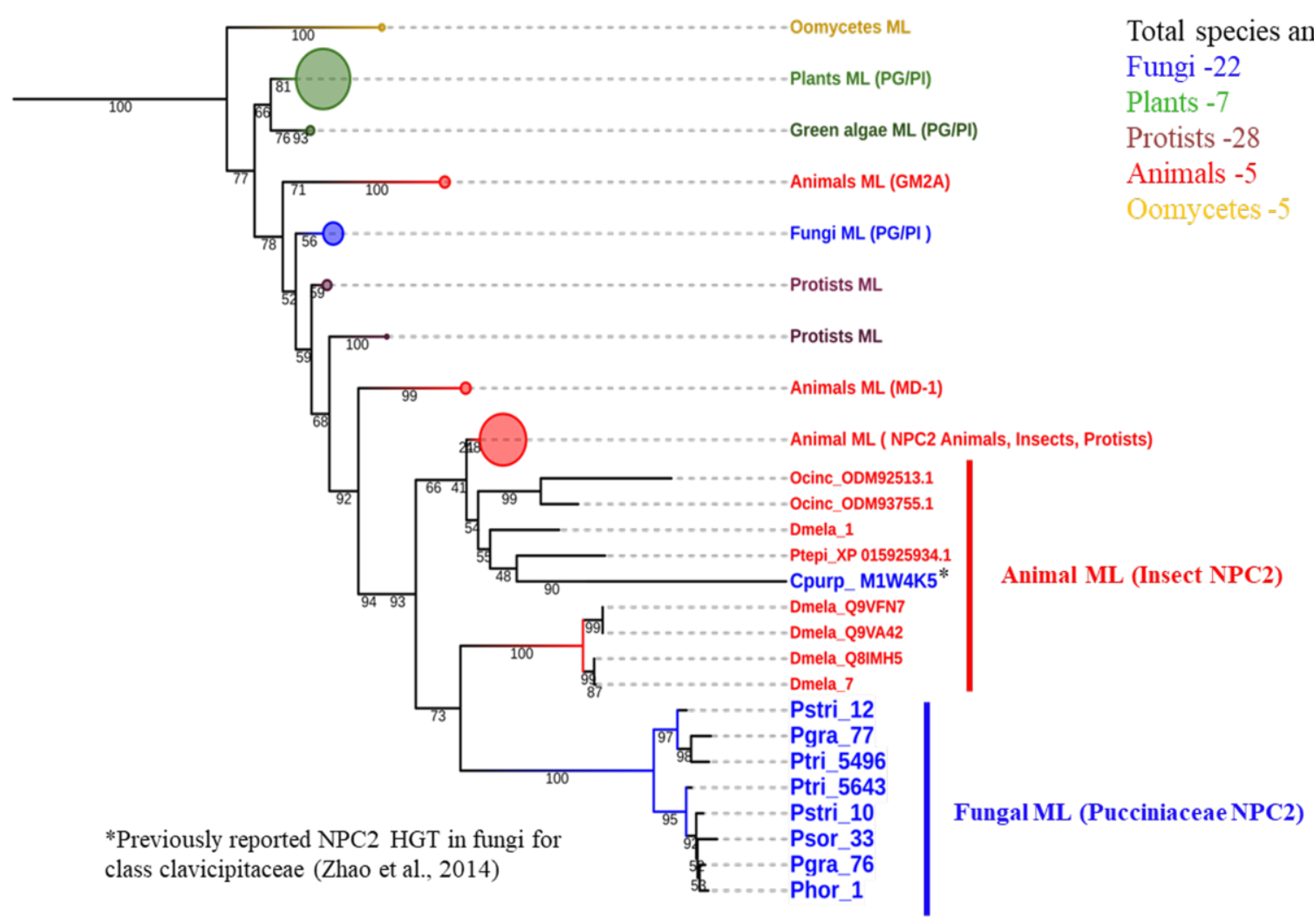

Fig. 3 


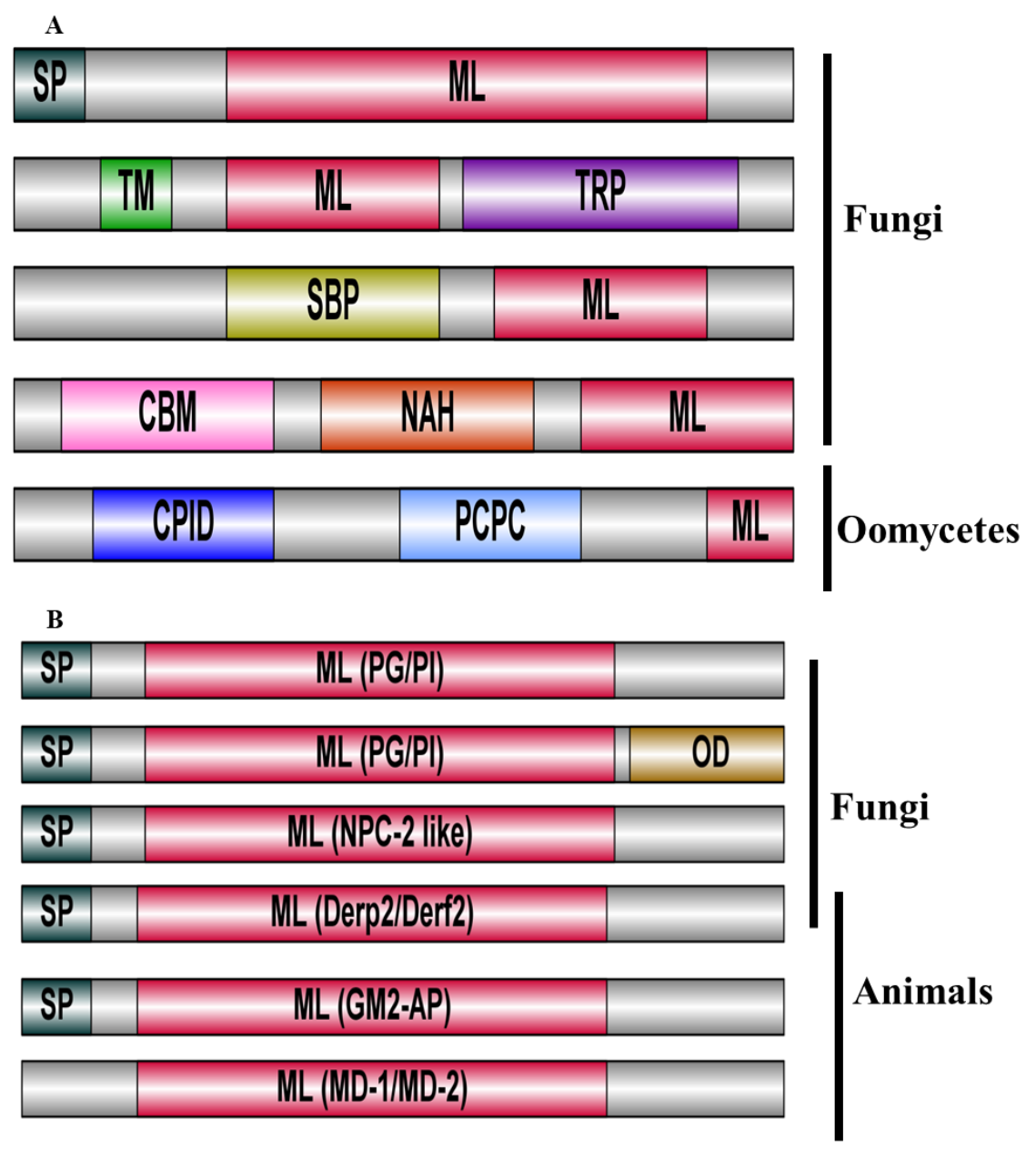

SP- Signal Peptide

ML- ML domain

TM- Transmembrane domain

TRP-Transient Receptor potential SBP-SKP1/BTB/POZ domain

CBM-Carbohydrate Binding

Module

NAH-Nucleophile

Aminohydrolases

CPID-Cathepsin Propeptide Inhibitor domain

PCPC-Peptidase C1A papain

SP- Signal Peptide

ML- ML domain

OD- Other Domains

ML (NPC-2 like) -NPC-2 like domain

ML (Derp2/Derf2)- Derp2/Derf2

like domain

ML (GM2-AP)- GM2 Ganglioside Activator

ML (MD-1/MD-2)- Myeloid

differentiation 1/Myeloid

differentiation 2 
Fig. 4.

$\mathbf{A}$

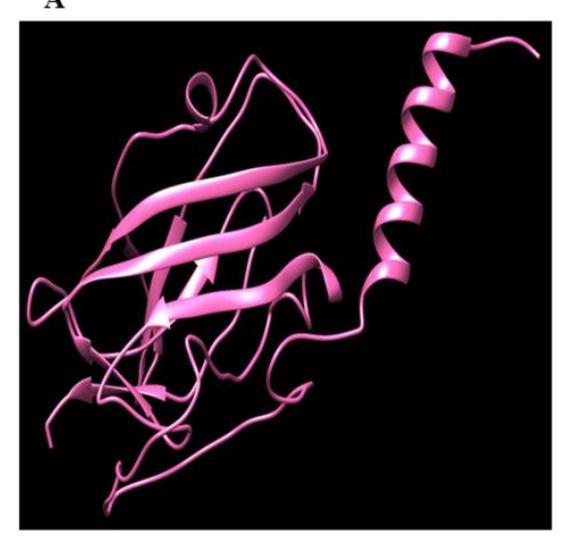

D

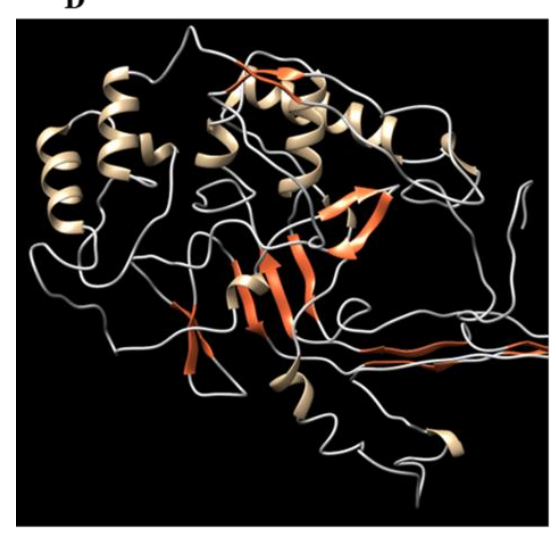

B

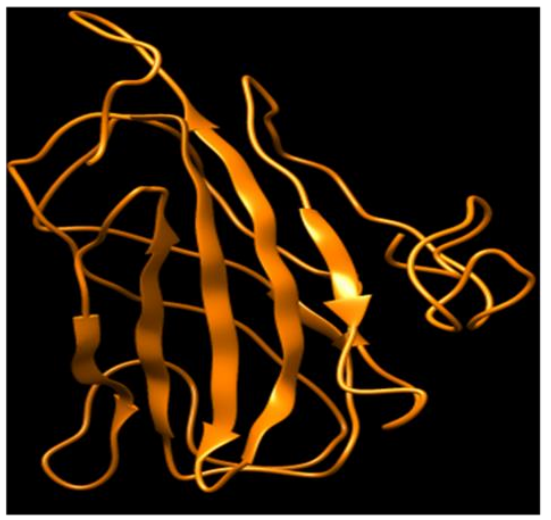

$\mathbf{E}$

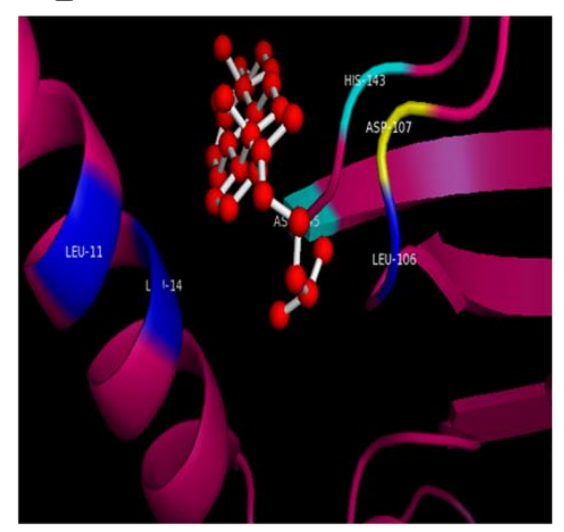

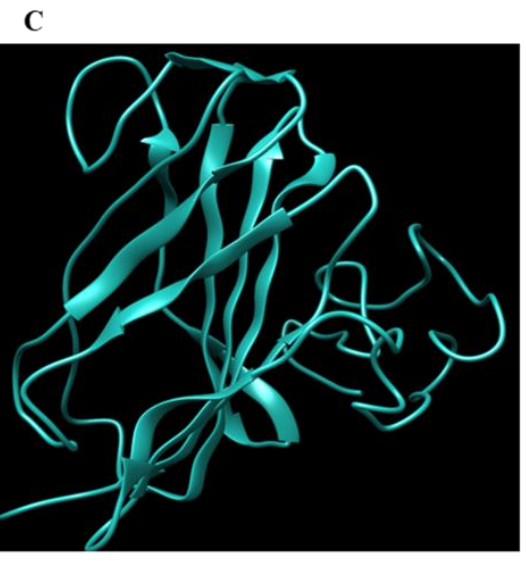

F

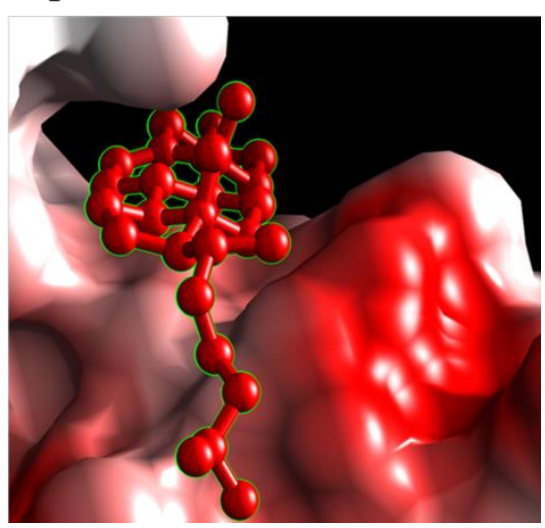

Fig. 5 

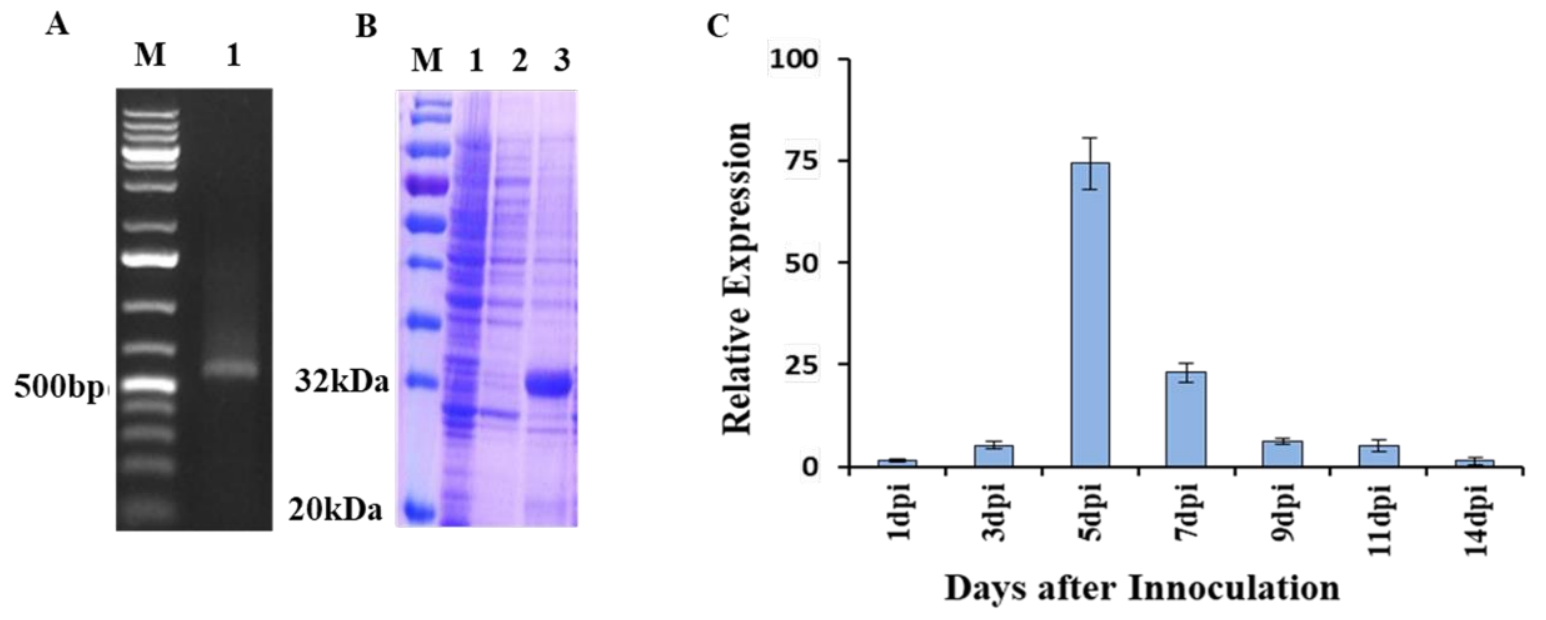

D
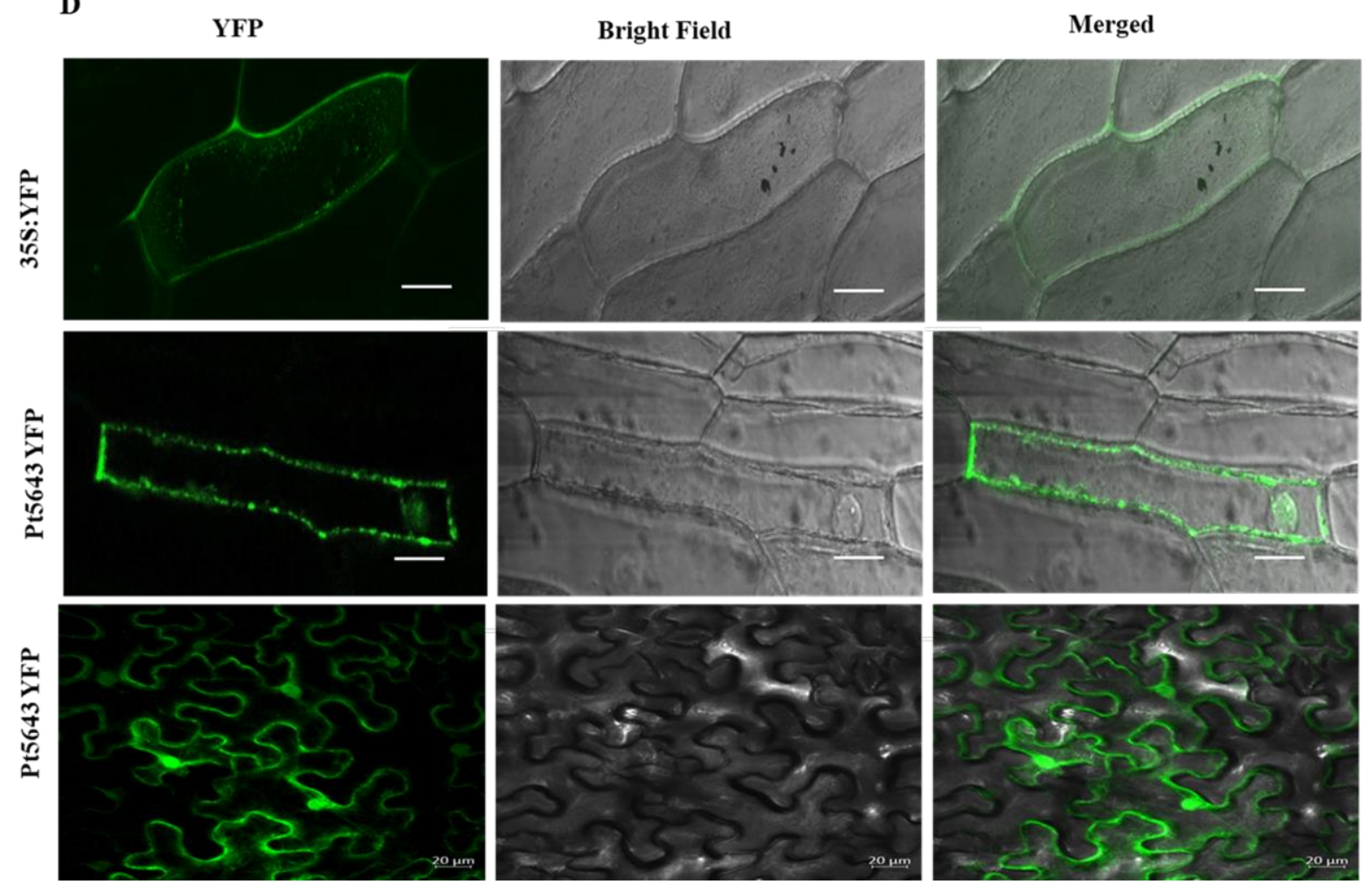

Fig. 6 


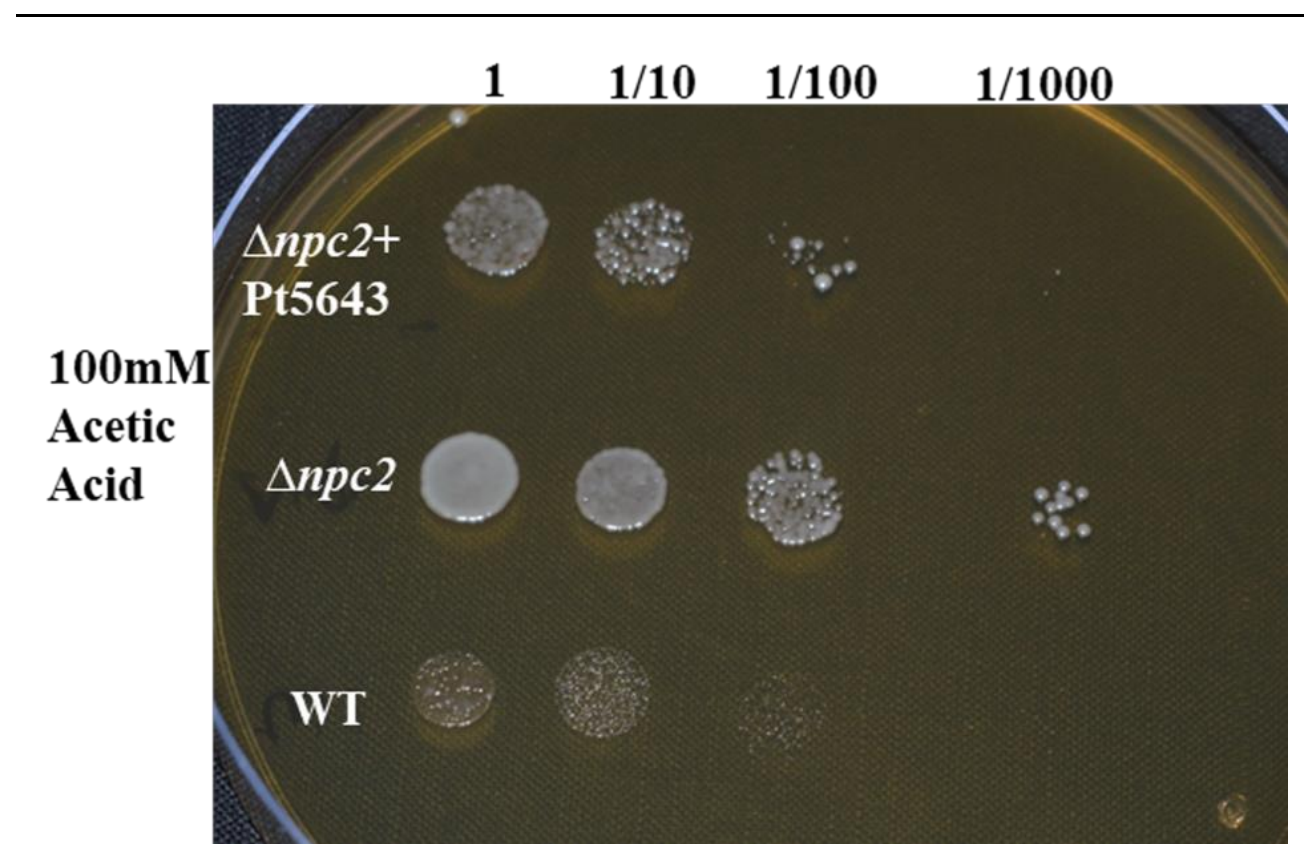


Fig. 7

A

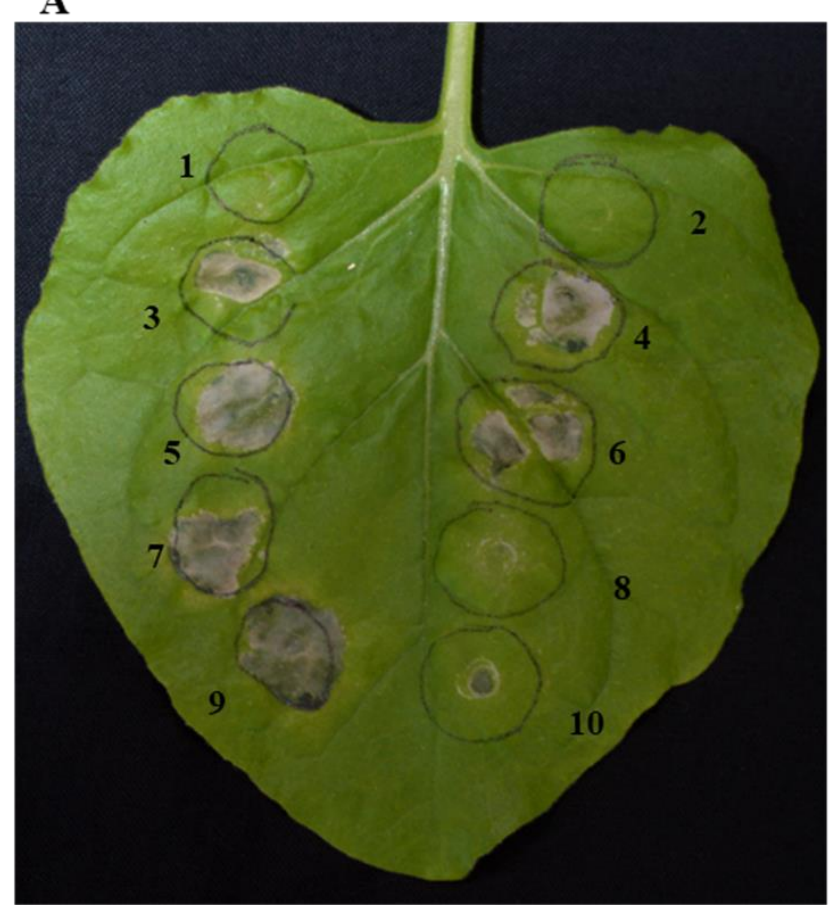

B

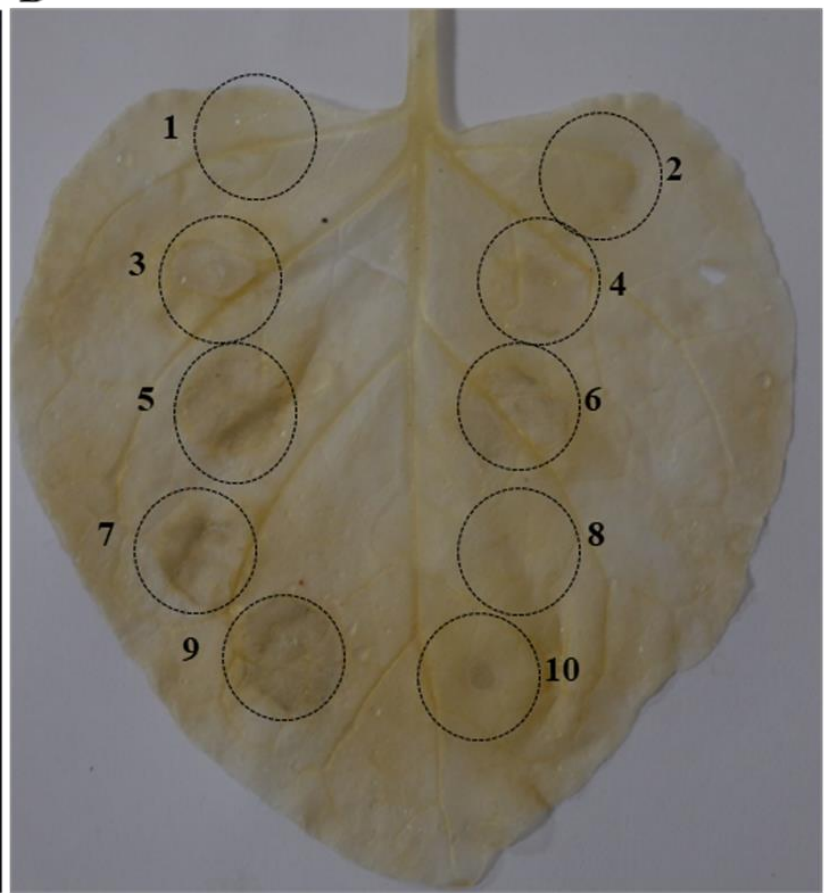


Fig. 8

Wild type

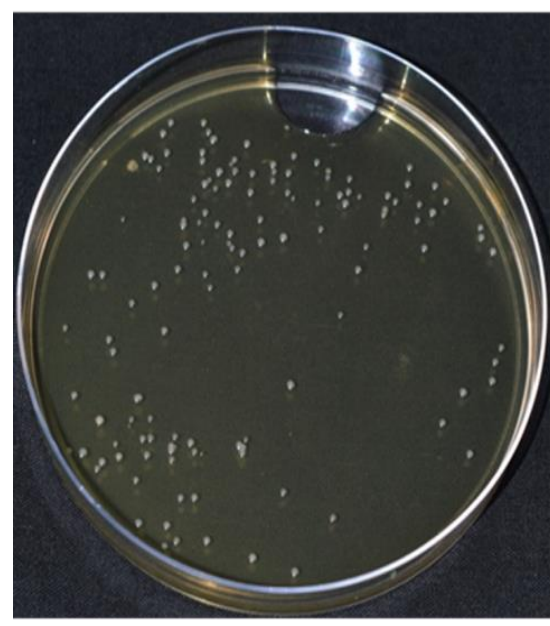

$n p c 2$ mutant

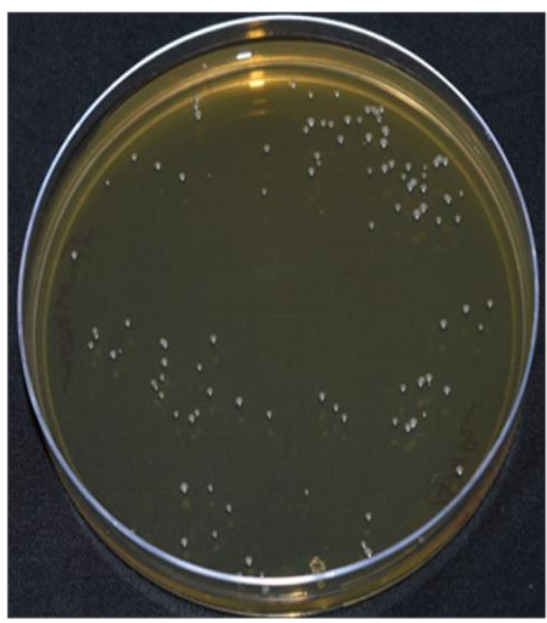

$n p c 2$ mutant $+\mathrm{Pt5643}$

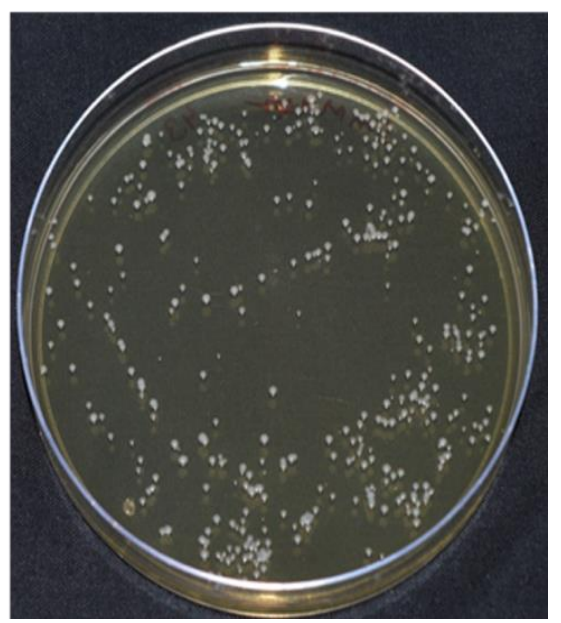


Table. 1. Positively Selected gene pairs of ML gene family in fungal species

\begin{tabular}{llllc}
\hline Gene1 & Gene2 & Ks & Ka & Ka/Ks \\
\hline BGNIJHo1 & BGN1JJF5 & 1.11 & 2.38 & 2.13 \\
BGAOAO78N & BGN1JJF5 & 2.47 & 3.10 & 1.26 \\
PSTRI 1O & PSTRI12 & 1.29 & 1.72 & 1.33 \\
U9SUS7_RI15 & U9SSM5_RI4 & 0.0656 & 0.1035 & 1.57 \\
\hline
\end{tabular}


Table 2. Molecular docking analysis of selected ML domain proteins from fungal species with various ligands

\begin{tabular}{|c|c|c|c|c|}
\hline $\begin{array}{l}\text { Organism } \\
\text { Name }\end{array}$ & Gene Name & Ligand & $\begin{array}{l}\text { Est. Free } \\
\text { Energy of } \\
\text { Binding } \\
\text { kcal/mol }\end{array}$ & $\begin{array}{l}\text { Total Intermole } \\
\text { Energy(kcal/me }\end{array}$ \\
\hline P. triticina & $P t \_5643$ & Zymosterol & -6.43 & -7.60 \\
\hline P. triticina & $P t \_5643$ & Cholesterol & -6.52 & -7.79 \\
\hline P. triticina & $P t \_5643$ & GP & -4.27 & -6.20 \\
\hline P. triticina & Pt_5643 & Lanosterol & -6.43 & -7.93 \\
\hline P. triticina & $P t \_5643$ & Lathosterol & -6.30 & -8.10 \\
\hline P. triticina & $P t \_5643$ & IPP & -2.14 & -7.81 \\
\hline P. triticina & $P t \_5643$ & Fungisterol & -6.49 & -8.05 \\
\hline$P$ triticina & $P t \_5643$ & Sequalene & -4.98 & -8.84 \\
\hline P. triticina & Pt_5643 & FPP & -4.25 & -7.02 \\
\hline P.triticina & Pt_5643 & Stigmast -7 enol & -0.27 & -2.87 \\
\hline P. triticina & Pt_5643 & $\mathrm{P}_{3} \mathrm{P}$ & -1.74 & -4.07 \\
\hline C. pupurea & $C p M 1 W A 48$ & Ergosterol & -10.03 & -11.15 \\
\hline C.pupurea & $C p M 1 W A 48$ & Sequalene & -8.35 & -11.85 \\
\hline C.pupurea & $C p M 1 W A 48$ & PS & -4.22 & -6.94 \\
\hline C.pupurea & $C p M 1 W A 48$ & FPP & $-7 \cdot 35$ & -9.89 \\
\hline C. pupurea & $C p M 1 W A 48$ & $\mathrm{P}_{3} \mathrm{P}$ & $-5 \cdot 51$ & -8.52 \\
\hline C. pupurea & CpM1WA48 & Cholestrol & -9.79 & -11.26 \\
\hline C. pupurea & $C p M 1 W A 48$ & 24 ethldiene lophenol & -10.57 & -11.89 \\
\hline C. pupurea & $C p M 1 W A 48$ & Lanosterol & -10.12 & -11.45 \\
\hline U. maydis & UMo4733 & Desmosterol & -7.24 & -8.56 \\
\hline U.maydis & UMo4733 & Fecosterol & -6.67 & -8.30 \\
\hline U. maydis & UMo4733 & Ergosterol & -6.43 & -7.86 \\
\hline U.maydis & UMo4733 & Lanosterol & $-5 \cdot 43$ & -8.41 \\
\hline U.maydis & UMo4733 & $\mathrm{P}_{3} \mathrm{P}$ & -2.68 & -4.82 \\
\hline
\end{tabular}




\begin{tabular}{|c|c|c|c|c|}
\hline P. sorghi & Psorghi_33 & Cholesterol & -7.44 & -8.96 \\
\hline P. sorghi & Psorghi_33 & Lanosterol & -5.95 & -10.49 \\
\hline P. sorghi & Psorghi_33 & Fungisterol & -8.05 & -10.61 \\
\hline P. sorghi & Psorghi_33 & Ergosterol & -6.45 & -7.88 \\
\hline P.sorghi & Psorghi_33 & FPP & -2.54 & $-5 \cdot 79$ \\
\hline B. graminis & BGAOAO78N1M3 & Cholesterol & -6.49 & -9.94 \\
\hline B.graminis & ВGAOAO78N1M3 & Fungisterol & $-5 \cdot 95$ & -10.61 \\
\hline $\begin{array}{l}\text { R.irregularis } \\
\text { R.irregularis }\end{array}$ & $\begin{array}{l}U 9 U 013 \_R I \\
U 9 U 013 \_R I\end{array}$ & Cholesterol & -9.05 & -10.68 \\
\hline R.irregularis & U9UO13_RI & Brassicasterol & -9.04 & -10.56 \\
\hline
\end{tabular}

${ }^{*}$ GP- Geranyl pyrophosphate, IPP- Isopentenyl pyrophosphate, FPP-Farnesyl pyrophosphate 